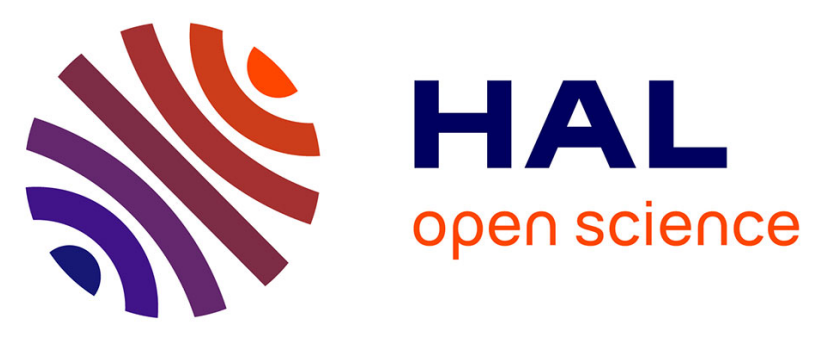

\title{
Numerical simulation of three-dimensional thermo-solutal convection of micropolar multi-walled carbon nanotubes water nanofluid stabilized by lignin and sodium polycarboxylate
}

Nessrin Manaa, Awatef Abidi, Patrice Estellé, Mohammed Naceur Borjini

\section{To cite this version:}

Nessrin Manaa, Awatef Abidi, Patrice Estellé, Mohammed Naceur Borjini. Numerical simulation of three-dimensional thermo-solutal convection of micropolar multi-walled carbon nanotubes water nanofluid stabilized by lignin and sodium polycarboxylate. Journal of Thermal Analysis and Calorimetry, 2022, 147 (4), pp.2985-3005. 10.1007/s10973-021-10667-9 . hal-03186405

\author{
HAL Id: hal-03186405 \\ https://hal.science/hal-03186405
}

Submitted on 31 Mar 2021

HAL is a multi-disciplinary open access archive for the deposit and dissemination of scientific research documents, whether they are published or not. The documents may come from teaching and research institutions in France or abroad, or from public or private research centers.
L'archive ouverte pluridisciplinaire HAL, est destinée au dépôt et à la diffusion de documents scientifiques de niveau recherche, publiés ou non, émanant des établissements d'enseignement et de recherche français ou étrangers, des laboratoires publics ou privés. 


\title{
Numerical simulation of three-dimensional thermo-solutal convection of micropolar multi-walled carbon nanotubes water nanofluid stabilized by lignin and sodium polycarboxylate
}

\author{
Nessrin Manaa ${ }^{a}$, Awatef Abidi ${ }^{\text {b,c,d }}$, Patrice Estellée ${ }^{\text {** }}$, Mohammed Naceur Borjini ${ }^{\text {a }}$ \\ ${ }^{a}$ Research Laboratory of Metrology and Energy Systems, National Engineering School, \\ Energy Engineering Department, Monastir University, Monastir City, Tunisia. \\ ${ }^{\mathrm{b}}$ Physics Department, College of Sciences Abha, King Khalid University, Saudi Arabia. \\ ${ }^{c}$ Research Laboratory of Metrology and Energy Systems LR81ES21, National Engineering \\ School, Energy Engineering Department, Monastir University, Monastir City, Tunisia. \\ ${ }^{\mathrm{d}}$ Higher school of Scienecs and Technology of Hammam Sousse, Sousse University, Tunisia. \\ e Univ Rennes, LGCGM, F-35000, Rennes, France.
}

Corresponding author: Patrice Estellé: patrice.estelle@univ-rennes1.fr 


\begin{abstract}
:
A computational analysis has been performed in this study to solve three-dimensional thermo-solutal natural convection in a differentially heated cubical enclosure filled with micropolar CNT-water nanofluid stabilized by two types of surfactants lignin and sodium polycarboxylate. The work is carried out for different pertinent parameters as Rayleigh number $\left(10^{4} \leq R a \leq 10^{6}\right)$, micropolar parameter $(0 \leq K \leq 10)$, buoyancy ratio $(-1 \leq N \leq 0)$ and nanoparticles's volume fraction $(0.0055 \% \leq \varphi \leq 0.557 \%)$.It is observed that the heat and mass transfer rates are lower for a micropolar nanofluid model when compared to the pure nanofluid model. In fact, the enhancement of micropolar parameter results a decrease of average Nusselt and Sherwood numbers. The use of lignin as a surfactant ameliorates heat and mass transfer rate and nanofluid flow better than the use of sodium polycarboxylate as a surfactant. The nanoparticles volume fraction can be used as a control element for heat rate and fluid flow. Thus, for a nanoparticles volume concentration less than the critical value, the flow intensity is ameliorated and is deteriorated when it exceed this value.
\end{abstract}

Keywords: thermo-solutal convection, three-dimensional, micropolar CNT-water nanofluid, surfactant.

\title{
Introduction
}

Over the last years, the demand on energy is become more intensive due to the extensive growth of industry and population. In fact, energy is considered as essential element for human being because her daily activities which need energy. The one of major energy which is needed in consuming process in industrial applications is double diffusive natural convection. It exist many applications in industry and research where the phenomena of double diffusive natural convection is present such as the crystal growth for the manufacture of semiconductors and electronics equipments cooling, solar collectors, home ventilation and heat exchangers. Moreover, utilizing nanofluid is one of the new methods for conservation energy during natural convection. These fluids are manufactured by the dispersion of nanoparticles in conventional heat transfer fluids. Itexists many types of nanoparticles used in nanofluids that differ in nature, such as metallic ( $\mathrm{Al}, \mathrm{Cu}, \mathrm{Ag} . .$.$) , oxide \left(\mathrm{Al}_{2} \mathrm{O}_{3}, \mathrm{TiO}_{2}, \mathrm{CuO} \ldots\right)$ and carbon-based materials. Putra et al. [1] investigated experimentally the natural convection of nanofluid in a horizontal cylinder filled with two types of nanoparticles $\mathrm{CuO}$ and $\mathrm{Al}_{2} \mathrm{O}_{3}$. They obtained a decrease in heat transfer rate with the enhancement in nanoparticles' concentrations. Khanafer et al. [2] carried out a numerical investigation on natural convection with $\mathrm{Cu}$ nanofluid and noticed heat transfer increase with an increase in nanoparticles' 
volume fraction. Jou and Tzeng [3] numerically examined the natural convection in a twodimensional enclosure, filled by $\mathrm{Cu}$ /water nanofluid for various pertinent parameters such as Rayleigh number, aspect ratio, buoyancy parameter and nanoparticles's volume fraction. Enhancement in heat transfer was shown to be more efficient with nanofluid than with pure fluid. Abu Nada et al. [4] carried out numerical investigations considering different nanofluids based on $\mathrm{Al}_{2} \mathrm{O}_{3}, \mathrm{TiO}_{2}, \mathrm{Cu}$ and $\mathrm{Ag}$, in horizontal annuli. They reported the dependence of heat transfer performance following the type of nanofluid used. Öztop and Abu Nada [5] carried a numerical investigation of heat transfer and fluid flow due to buoyancy forces in a partially heated enclosure filled with different types of nanofluids. They found that the average Nusselt number increases with the nanoparticles volume fraction.A numerical study of forced convective heat transfer in horizontal tubes filled with $\mathrm{Al}_{2} \mathrm{O}_{3}$ /water nanofluid was investigated by Minea [6]. The author established that the heat transfer coefficient of water-based $\mathrm{Al}_{2} \mathrm{O}_{3}$ nanofluids is increased compared to that of pure water. Bouhalleb and Abbassi [7] numerically studied the natural convection of $\mathrm{CuO}$-water nanofluid in inclined cavity differentially heated. They showed that the efficiency of heat transfer is enhanced by the addition of nanoparticles into base fluid. However, there is acritical solid volume fraction which maximizes the heat transfer rate. Minea et al. [8] carried out a benchmark study on the numerical approach in nanofluids' simulation. They compared different numerical approaches with experimental studies, concluding that multiphase model gives the most reliable results for nanofluid flow in long straight pipe. Purusothaman et al. [9] carried out a numerical analysis aboutnatural convective heat transfer ina three dimensional rectangular enclosure filled with nanofluid and in the presence of an array of discrete heaters. They founded that the averaged Nusselt number increases with increase in both the Rayleigh number and the nanoparticle solid volume fraction. Selimefendigil and Öztop [10] investigated numerically the three dimensional mixed convection in a cubic enclosure with two rotating cylinders and containing various types of nanofluids. The average heat transfer is enhanced with nanoparticles, the higher rate being achieved for $\mathrm{Cu}$-water nanofluid compare to $\mathrm{Al}_{2} \mathrm{O}_{3}$-water nanofluid and $\mathrm{TiO}_{2}$-water nanofluid. A numerical study on laminar double diffusive natural convection in a square cavity was performed by Esfahani and Bordbar [11]. Different types of nanofluid were used and the effect of pertinent parameters (Rayleigh number, Lewis number, buoyancy fraction and nanoparticles volume fraction) on heat and mass transfer rates was explored. Also, Parvin et al. [12] numerically investigated double-diffusive natural convection in a partially heated enclosure filled with $\mathrm{Al}_{2} \mathrm{O}_{3}$ /water nanofluid. It is noticed that the Rayleigh number and nanoparticles volume fraction influence on the distributions of 
isotherms and iso-concentrations. Chen et al. [13] thoroughly discussed the influence of nanoparticles' volume fraction on Nusselt and Sherwood numbers for both laminar and turbulent regimes. In fact, they concluded that the effects adding nanoparticles into base fluid are totally differentbetween the laminar regimes and turbulent regimes. Recently, a study was conducted by Aly and Raizah [14] on double diffusion and mono diffusion natural convection with nanofluids using incompressible smoothed particle hydrodynamics method. They founded that the increase Soret number accompanied by a decrease in Dufour number result in an enhancement inheat transfer rate and a reduction in mass transfer rate.Among all nanoparticles available for producing nanofluids, carbon nanotubes (CNT) are more recommending than other traditional nanoparticles because of their relatively low density and very high thermal conductivity.Wen and Ding [15] studied the effective thermal conductivity of aqueous suspensions of MWCNTs. They used the sodium polycarboxylate as surfactant to stabilize the nanofluids. They obtained an enhancement of thermal conductivity when the nanoparticles volume concentrationincreases, and the dependence was nonlinear even at very low concentrations. They founded that an enhancement in temperature increases the effective thermal conductivity of nanofluid. Jiang el al.[16] investigated the characteristics of CNT nano-refrigerants and their thermal conductivity. They founded that the thermal conductivities of CNT nanorefrigerants are much higher than those of CNT-water nanofluids and they increase significantly with the increase of the nanoparticles volume fraction. Halelfadl et al. [17] discussed the optimization of a rectangular microchannel filled with aqueousmulti-walled carbon nanotubes (MWCNTs)/water nanofluid as coolant. They showed that using nanofluid induces a decrease of the total thermal resistance and leads to an enhancement of thermal performances at high temperatures. Estellé et al. [18] investigated the thermal conductivity of carbon nanotube water-based nanofluids. They studied the effect of the volume fraction of nanoparticles, temperature, nanoparticles aspect ratio and different types of surfactant. They observed an enhancement of thermal conductivity of nanofluid at low volume fraction. However, this enhancement is weakly affected by the carbon nanotubes aspect ratio and the type of surfactant used. A study of the effect of lignin as surfactant on viscosity and thermal conductivity of CNT/water-based nanofluids was investigated by Estellé et al. [19]. They compared the effect of lignin on the viscosity and thermal conductivity of CNT/water nanofluid with the effect of sodium polycarboxylate. They noticed that the thermal conductivity of nanofluid increase with the nanoparticles volume concentration. Rahman et al. [20] numericallyinvestigated the natural convective heat transfer and fluid flow in a square cavity filled with CNT-water nanofluid with non-isothermal heating. They founded that there 
is an optimum value for nanoparticles volume fraction to control heat transfer, temperature distribution and flow field. Job and Gunakala [21] investigated the unsteady MHD natural convection flows of two types of nanofluid $\mathrm{Al}_{2} \mathrm{O}_{3}$ /water and SWCNT/water within a symmetrical wavy trapezoidal enclosure with viscous and Joule dissipation effects. They studied the effect of time, Hartmann number, wall amplitude, and solid volume fractionon the fluid flow and heat transferrate. They showed that the SWCNT/water nanofluid has a higher heat transfer rate at the wavy bottom wall than the $\mathrm{Al}_{2} \mathrm{O}_{3}$ /water nanofluid. Estellé et al. [22] investigated theoreticallythe natural convection in a square cavity partially heated filled with CNT water-based nanofluids. They analyzed the effect of the average temperature and the nanoparticles volume fraction, driving temperature between hot and cold walls and role of surfactant. They founded that the Nusselt number of nanofluids is decreased with the increase of the volume fraction of nanofluid, which is related to non-Newtonian behavior of nanofluids. Minea and Estellé [23] carried out a numerical study of the heat transfer behavior of carbon nanotubes nanofluids under laminar forced flow in horizontal pipe. They evidenced that the convective heat transfer coefficient of nanofluid is better for low Re number and higher nanoparticles volume fraction. Kolsi et al. [24] performed a computational analysis about an open cubic cavity filled with CNT-water nanofluid. This numerical analysis is performed for different governing parameters as Rayleigh number, nanoparticles volume fraction, Hartmann number and inclination angle of the fin. It is concluded that heat transfer rate and fluid flow dependsonthese parameters. Al-Rashed et al. [25] carried out a numerical study of entropy generation inside an inclined cubical cavity differentially heated filled with CNT/water nanofluid. They analyzed the effects of Rayleigh number, nanoparticles volume fraction, inclination angle of cavity and thermal conductivity ratio on entropy generation. Rahimi et al. [26] conducted a numerical and experimental analysis on heat transfer performance of 3D natural convection in a cubic cavity filled with double-walled CNTs-water nanofluid at different temperature differences between side hot and cold walls. In this work, the thermo-physical properties such us the thermal conductivityand the dynamic viscosity, of the double-walled CNTs-water nanofluid are experimentally evaluated. They revealed that the changein temperature difference influences the temperature distribution within the cavity. However, the enhancement of nanoparticles volume concentration has no significant effect on the temperature. Also, they demonstrated that it exist an optimum value of solid volume fraction forhighest value of average heat transfer coefficient and Nusselt number. Over this optimum,both parameters are deteriorated. 
The theory of Enrigen [27] can be used to elucidate fluid particles' micro-motions which cannot be explained by the classical models. In fact, physically, fluid particles may expand, contract, rotate about their own axis or may even change their shape due to the shear stress applied on them. So, the micropolar fluid theory can be valuable to explore fluid behavior of polymer fluids, solidification of liquid crystals, cooling of a metallic plate in a bath, exotic lubricants, colloidal systems, and biological fluids, for which the theory of Navier-Stokes is inadequate to describe the impacts of the microstructures on fluid motion.

Description and applications of natural convection with micropolar fluid in enclosures have been studied in the following papers. Aydin and Pop [28] performed a numerical study of a steady natural convective heat transfer of micropolar fluids in a square cavity. They identified that an increase of the micropolar parameter reduced the heat transfer rate. Zadravecet al. [29] carried out a numerical investigation of natural convection of a micropolar fluid within an enclosure. It was obtainedthat the local and average Nusselt numbers decrease with the enhancement of the micropolar parameter. Jena et al. [30] extracted the numerical solution of the transientbuoyancy-opposed double diffusive natural convection of a micropolar fluid. They observed that the flow strength and heat and mass transfer rates are deteriorated for growing values of the micropolar parameters. Abidi and Borjini [31] discussedthe effect of micro-constituents in micropolar fluid on the three-dimensional doublediffusive natural convection. They evidenced the deterioration in three-dimensional character of the flow with a considerable reduction in heat and mass transfer rates with an increase in the micropolar parameter.

The rotating micro-constituents' effects in nanofluids should be judiciously exploited to understand the behavior of fluid flow effectively. Bourantas and Loukopoulos [32] theoretically examined the natural convection of micropolar nanofluids. One of the main objectives of this work wasto study the effect of the micropolar model on heat transfer rate. They founded that the increase in nanoparticles micro-rotation caused a decrease in the heat transfer rate. Hashemi et al. [33] numerically investigated micropolarCu/water nanofluid natural convection. A heat generation source in a porous medium was considered in this case. They highlighted that the rotational strength of vortices enhances with the increase in the micropolar parameter. Hussanan et al. [34] used five types of oxide nanoparticles dispersed in water, kerosene and engineoilto explore the unsteadynatural convection flow of micropolar nanofluids past a vertical plate. They obtained that the type of nanoparticles and the base fluid significantly influence heat transfer rate and flow behavior.The effect of the magnetic field on natural convection in a micropolar nanofluid was investigated by Bourantas and Loukopoulos 
[35]. The study focused on an inclined rectangular cavity and reveals that the presence of nanoparticles influences the thermal properties of the base fluid.The mixed convection in a lid-driven cavity filled with micropolar nanofluid was analyzed by Ahmed et al. [36].They founded that the average Nusselt number along the heat source increases when the solid volume fraction increases. Nering and Rup [37] numerically analyzed the heat transfer of micropolar nanofluids with two different base fluids: ethylene glycol and water. They obtained that the maximum heat transfer enhancement parameter for nanofluid without micropolar properties appears for $\mathrm{Cu} /$ ethylene glycol nanofluid. On the other hand, they observed that $\mathrm{Al}_{2} \mathrm{O}_{3} /$ water nanofluid has a greater value of heat transfer enhancement. Izadi et al. [38] studied the natural convection of a micropolar nanofluid inside a porous cavity with the use of a thermal non-equilibrium model. They founded that the increase of micropolar parameter induces a reduction of the thermal resistance of the fluid phase. Rashad et al. [39] reported the outcomes of a computational investigation on micropolar nanofluid natural convection inside an enclosure with a heatingsource located at the bottom. Their results indicate that an increase in the micropolar parameter causes a reduction in the local Nusselt number. Also they concluded that as the nanoparticle volume fraction increases, the rate of heat transfer increases. Abidi et al. [40] carried out the 3D numerical simulation of both heat and mass transfer rates and fluid flow in a cubic cavity filled with an $\mathrm{Al}_{2} \mathrm{O}_{3}$ /water micropolar fluid. The simulations are conducted under a uniform magnetic field. The results reveal that heat and mass transfer rates and three-dimensional characters of the flow are weaker when the micropolar nanofluid model was used compared to the pure nanofluid model. Manaa et al. [41] investigated the thermo-solutal natural convection of a micropolar nanofluid filled 3D enclosure with different types of nanoparticles $\left(\mathrm{Al}_{2} \mathrm{O}_{3}, \mathrm{TiO}_{2}, \mathrm{Cu}\right.$ and $\left.\mathrm{Ag}\right)$ considering the effect of relevant parameters on heat and mass transfer characteristics. They obtained that both heat and mass transfer rates and the three-dimensional character of the flow for the micropolar nanofluid model are smaller compared with that of a pure nanofluid model. Their results show that the rates of heat and mass transfer decrease with an enhancement in micropolar parameter as well as nanoparticlesvolume fractions. Additionally, they concluded that the type of nanoparticles significantly influences heat and mass transfer rates. Manaa et al. [42] realized a numerical study about three-dimensional double-diffusive natural convection in a cubic cavity filled with $\mathrm{Cu}-\mathrm{Al}_{2} \mathrm{O}_{3} /$ water micropolar hybrid nanofluid. They compared the performance enhancement of $\mathrm{Cu}-\mathrm{Al}_{2} \mathrm{O}_{3} /$ water micropolar hybrid nanofluid with the $\mathrm{Cu} /$ water simple nanofluid. Besides, the influences of concentration of nanoparticles, Rayleigh number, buoyancy ratio, and micropolar parameter on the flow field and heat and 
mass transfer rates are analyzed. They founded that heat and mass transfer rates are lower for a micropolar nanofluid model when compared to the pure nanofluid model.

The main aim of this work is to perform a computation alanalysis on the three-dimensional double-diffusive natural convection in multi-walled CNT/water micropolar nanofluid stabilized with two types of surfactants lignin and sodium polycarboxylate. In this study, experimental thermophysical properties determined in previous works were used. The current work can be used to control and monitor heat and mass transfer and fluid flow in a cubic enclosure. The effect of the type of used surfactant to stabilize the micropolar nanofluid, micropolar parameter, nanoparticles' volume fraction, buoyancy ratio, and the Rayleigh number on heat and mass transfer rates and flow behavior are meticulously elucidated. The above literatures clearly show that there is no work in the literature on three-dimensional double diffusive natural convection for micropolar multi-walled CNT/water nanofluid. Thus, this is the novelty of the current work.

\section{Mathematical modeling}

A simple schematic view of the thermo-solutal natural convection problem under study is described in Fig. 1. The cube is filled with multi-walled CNT water-based micropolar nanofluid stabilized with two types of surfactants lignin and sodium polycarboxylate respectively. The two vertical walls parallel to the plane $(y-z)$ are subject to constant temperatures $\left(T_{H}>T_{C}\right)$ and constant concentrations $\left(C_{H}>C_{L}\right)$. The other walls are supposed to be impermeable and adiabatic. The dynamic and thermal slips between nanoparticles and the base fluid are negligible. During the process of the natural convection, all the properties of the base fluid and nanoparticles are unchangeable except the density in the buoyancy term in momentum equation, its variations being modeled using Boussinesq approximation. The effects of Soret and Dufour are assumed to be negligible.

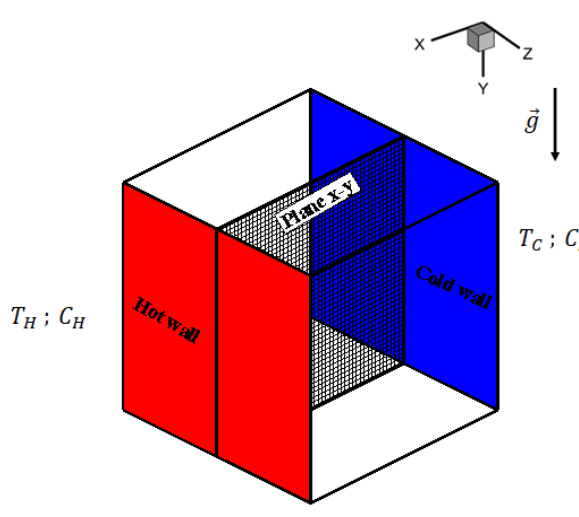

(a)

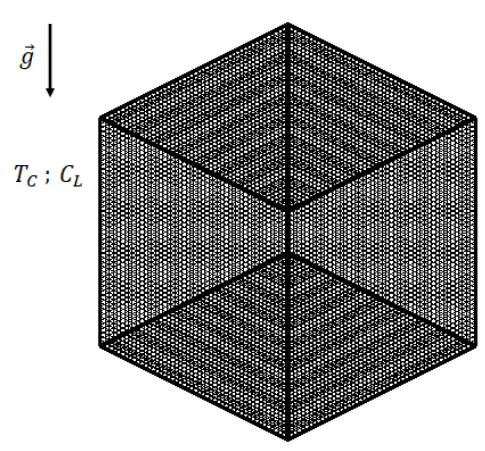

(b)

Fig.1 (a) Schematic of the considered problem, (b) Mesh surfaces 


\section{Thermophysical properties of multi-walled carbon nanotube nanofluid}

For comparison purpose with nanofluids, water which is the base fluid of nanofluids was taken as a reference. The thermo-physical properties of water used for the simulations are reported in [43]. The density, the heat capacity, the thermal conductivity, the thermal expansion coefficient and the dynamic viscosity of water are respectively equal to $998.205 \mathrm{~kg}$ $\mathrm{m}^{-1}, 4182 \mathrm{~J} \mathrm{~kg}^{-1} \mathrm{~K}^{-1}, 0.6 \mathrm{~W} \mathrm{~m}^{-1} \mathrm{~K}^{-1}, 0.00021 \mathrm{~K}^{-1}$ and $1.003 \mathrm{mPa} \mathrm{s}$.

In this study, it is considered carbon nanotubes with density of $1800 \mathrm{~kg} \mathrm{~m}^{-3}$ and purity of $90 \%$ and $9.2 \mathrm{~nm}$ and $1.5 \mu \mathrm{m}$ in average diameter and length, respectively, dispersed in a mixture of water and surfactant. To improve the dispersion and stability of multi-walled CNT within water and to reduce clogging and the sedimentation with time, it was used two types of surfactants lignin and sodium polycarboxylate. The preparation of nanofluid was reported in $[18,19,22]$. The volume fraction of carbon nanotubes under consideration ranges from 0.0055 to $0.557 \%$. Density, thermal conductivity, viscosity at $20^{\circ} \mathrm{C}$ were experimentally determined in $[18,19,22]$ from well-designed procedures while heat capacity was theoretically determined neglecting heat capacity of surfactants. All these results are shown in Fig. 2 and used in the present numerical simulations.

In the following, nanofluids produced with lignin are labeled N2. N3 is used for nanofluids obtained with sodium polycarboxylate as a surfactant. 


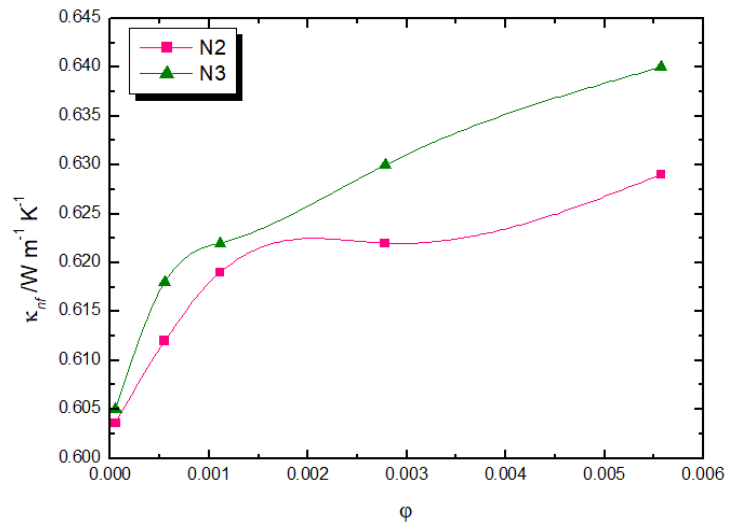

(a)

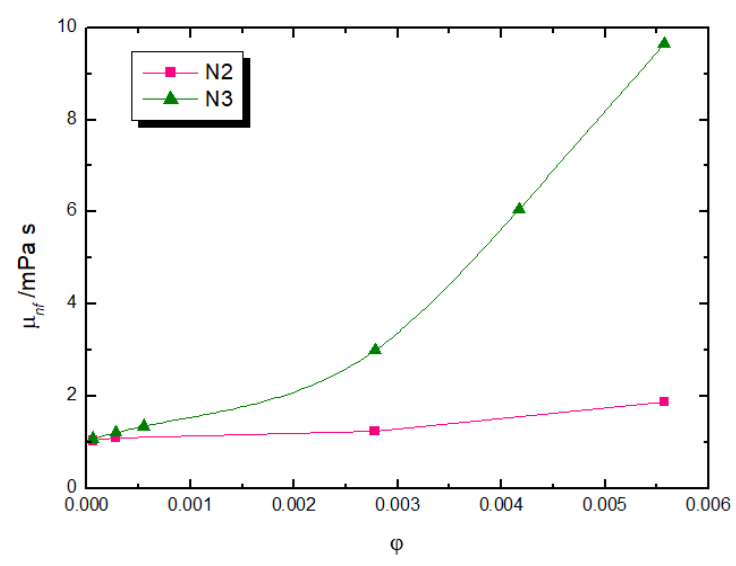

(c)

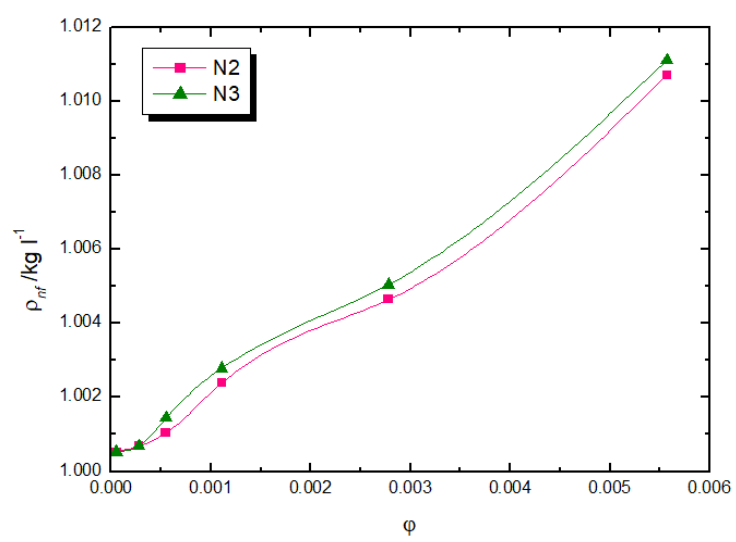

(b)

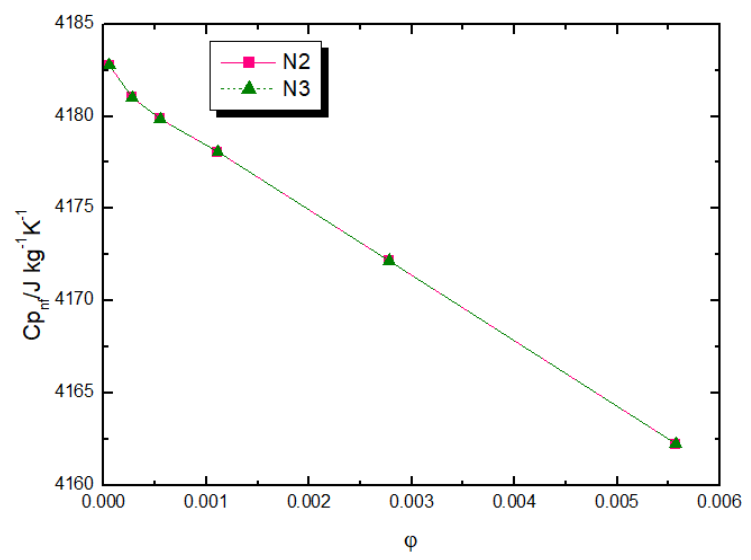

(d)

Fig. 2 Thermophysical properties of CNT nanofluids at $20^{\circ} \mathrm{C}$ with two types of surfactants:

(a) thermal conductivity, (b) density, (c) dynamic viscosity and (d) heat capacity

To make easier the implementation of thermophysical data of nanofluids in the numerical simulation and program [44], the following formulas of thermophysical properties of nanofluids have been adjusted on experimental data.

Table 1 Thermophysical properties of nanofluid at $\mathrm{T}=20^{\circ} \mathrm{C}$

\begin{tabular}{lcc}
\hline & $\mathrm{N} 2$ & $\mathrm{~N} 3$ \\
\hline Density/ kg m & $\rho_{n f}=18.4 \varphi+1000$ & $\rho_{n f}=19 \varphi+1000$ \\
\hline Thermal conductivity/ & $k_{n f}=0.0053 \operatorname{Ln}(\varphi)+0.63$ & $k_{n f}=0.0072 \operatorname{Ln}(\varphi)+0.64$ \\
$\mathrm{~W} \mathrm{~m}{ }^{-1} \mathrm{~K}^{-1}$ & +1.05074 & +1.176 \\
\hline $\begin{array}{l}\text { Dynamic viscosity/ } \\
\mathrm{mPa} \text { s }\end{array}$ & $\mu_{n f}=27849.4516 \varphi^{2}-9.2088 \varphi$ & $\mu_{n f}=28980 \varphi^{2}-82.18 \varphi$ \\
\hline Heat capacity/ $\mathrm{J} \mathrm{kg}^{-1}$ & $C p_{n f}=-38.38 \varphi+4182$ & $C p_{n f}=-38.38 \varphi+4182$ \\
\hline
\end{tabular}


The thermal expansion coefficient of nanofluids was calculated from the following equation [2] as:

$$
\beta_{n f}=\frac{(1-\varphi)(\rho \beta)_{f}+\varphi(\rho \beta)_{p}}{(1-\varphi) \rho_{f}+\varphi \rho_{p}}
$$

Where the thermal expansion coefficient of carbon nanotubes at $20^{\circ} \mathrm{C}$ was taken as $2.10^{-5}[22]$.

\section{Governing Equations}

The dimensionless governing equations are thefollowings:

$$
\begin{gathered}
\nabla \vec{U}=0 \\
\frac{\partial \vec{U}}{\partial t}+(\vec{U} . \nabla) \vec{U}=-\frac{1}{\rho_{n f}} \nabla P+\operatorname{Pr}\left(\frac{\mu_{n f}}{\mu_{f}}+K\right)\left(\frac{\rho_{f}}{\rho_{n f}}\right) \nabla \times \vec{U}- \\
\operatorname{Pr} K\left(\frac{\rho_{f}}{\rho_{n f}}\right) \nabla \times \vec{H}+\operatorname{RaPr}\left(\frac{\left(\rho \beta_{T}\right)_{n f}}{\left(\rho \beta_{T}\right)_{f}}\right)\left(\frac{\rho_{f}}{\rho_{n f}}\right)(T-N C) \\
\left(\frac{\partial \vec{H}}{\partial t}+(\vec{U} . \nabla) \vec{H}\right)=\operatorname{Pr}\left(\frac{\mu_{n f}}{\mu_{f}}+\frac{K}{2}\right)\left(\frac{\rho_{f}}{\rho_{n f}}\right) \nabla^{2} \vec{H}+\operatorname{Pr} K\left(\frac{\rho_{f}}{\rho_{n f}}\right)(\nabla \times \vec{U}-2 \vec{H}) \\
\frac{\partial T}{\partial t}+(\vec{U} . \nabla) T=\left(\frac{\left(\rho C_{P}\right)_{f}}{\left(\rho C_{P}\right)_{n f}}\right)\left(\frac{k_{n f}}{k_{f}}\right) \nabla^{2} T \\
\frac{\partial C}{\partial t}+(\vec{U} . \nabla) C=\frac{1}{L e}\left(\frac{\left(\rho C_{P}\right)_{n f}}{\left(\rho C_{P}\right)_{f}}\right)\left(\frac{k_{f}}{k_{n f}}\right) \nabla^{2} C
\end{gathered}
$$

The following non-dimensional variables are used for the normalization of the required governing equations (2) (3) (4) (5) and (6):

$$
\begin{aligned}
x=\frac{x \prime}{L}, y & =\frac{y^{\prime}}{L}, z=\frac{z \prime}{L}, t=\frac{\alpha t^{\prime}}{L^{2}},\left(U_{1}, U_{2}, U_{3}\right)=\frac{\left(U_{1}^{\prime} \cdot U_{2}^{\prime} \cdot U_{3}^{\prime}\right) L}{\alpha}, T=\frac{T^{\prime}-T_{C}^{\prime}}{T_{H}^{\prime}-T_{C}^{\prime}}, C=\frac{C^{\prime}-C_{L}^{\prime}}{C_{H}^{\prime}-C_{L}^{\prime}} \\
\operatorname{and}\left(H_{1}, H_{2}, 0\right) & =\frac{\left(H_{1}^{\prime}, H_{2}^{\prime}, 0\right) L^{2}}{\alpha}
\end{aligned}
$$

The vector of micro-rotation $\overrightarrow{H^{\prime}}$ may be presented as follows:

$\overrightarrow{H^{\prime}}=\left(\overrightarrow{H_{1}}, \overrightarrow{H_{2}}, 0\right)$ (Agarwal et al. [45], Takhar et al. [46], and Chamkha et al. [47]).

In these equations, the dimensionless $\operatorname{Pr}, L e, R a, N$ and $K$ numbers are respectively defined as

$$
\operatorname{Pr}=\frac{v_{f}}{\alpha_{f}}, \mathrm{~L} e=\frac{\alpha}{D}, R a=\frac{g \beta_{T f}\left(T_{H}-T_{C}\right) L^{3}}{v_{f} \alpha_{f}}, N=\frac{\beta_{C f}\left(C_{H}-C_{L}\right)}{\beta_{T f}\left(T_{H}-T_{C}\right)} \text { and } K=\frac{\mathrm{k}}{\mu_{f}}
$$


The vorticity-vector potential formulation is used in the present study to eliminate the pressure term and makes easier the numerical treatment. The vorticity and vector potential are respectively defined by the following two relations: $\vec{U}=\nabla \times \vec{\psi}$ and $\vec{\omega}=\nabla \times \vec{U}$.

The system of governing equationsof the phenomenonis

$$
\begin{gathered}
\nabla^{2} \vec{\psi}=-\vec{\omega} \\
\frac{\partial \vec{\omega}}{\partial t}+(\vec{U} . \nabla) \vec{\omega}-(\vec{\omega} . \nabla) \vec{U}=\operatorname{Pr}\left(\frac{\mu_{n f}}{\mu_{f}}+K\right)\left(\frac{\rho_{f}}{\rho_{n f}}\right) \nabla^{2} \vec{\omega}-\operatorname{Pr} K\left(\frac{\rho_{f}}{\rho_{n f}}\right) \nabla^{2} \vec{H} \\
+\operatorname{RaPr}\left(\frac{\left(\rho \beta_{T}\right)_{n f}}{\left(\rho \beta_{T}\right)_{f}}\right)\left(\frac{\rho_{f}}{\rho_{n f}}\right)\left(\left[\frac{\partial T}{\partial z}, 0,-\frac{\partial T}{\partial x}\right]-N\left[\frac{\partial C}{\partial z}, 0,-\frac{\partial C}{\partial x}\right]\right) \\
\left(\frac{\partial \vec{H}}{\partial t}+(\vec{U} . \nabla) \vec{H}\right)=\operatorname{Pr}\left(\frac{\mu_{n f}}{\mu_{f}}+\frac{K}{2}\right)\left(\frac{\rho_{f}}{\rho_{n f}}\right) \nabla^{2} \vec{H}+\operatorname{Pr} K\left(\frac{\rho_{f}}{\rho_{n f}}\right)(\vec{\omega}-2 \vec{H}) \\
\frac{\partial T}{\partial t}+(\vec{U} \cdot \nabla) T=\left(\frac{\left(\rho C_{P}\right)_{f}}{\left(\rho C_{P}\right)_{n f}}\right)\left(\frac{k_{n f}}{k_{f}}\right) \nabla^{2} T \\
\frac{\partial C}{\partial t}+(\vec{U} . \nabla) C=\frac{1}{L e}\left(\frac{\left(\rho C_{P}\right)_{n f}}{\left(\rho C_{P}\right)_{f}}\right)\left(\frac{k_{f}}{k_{n f}}\right) \nabla^{2} C
\end{gathered}
$$

The local Nusselt and Sherwood numbers on the isothermal walls are defined by:

$$
\begin{gathered}
\left.\left.N u=\frac{k_{n f} \frac{\partial T^{\prime}}{\partial x^{\prime}}}{k_{f} \frac{T^{\prime} H^{-} T^{\prime} C}{L}}\right)_{x^{\prime}=0,1}=-\frac{k_{n f}}{k_{f}} \frac{\partial T}{\partial x}\right)_{x=0,1} \\
\left.\left.S h=\frac{D \frac{\partial C^{\prime}}{\partial x^{\prime}}}{D \frac{C^{\prime} H^{-C^{\prime} L}}{L}}\right)_{x^{\prime}=0,1}=\frac{\partial C}{\partial x}\right)_{x=0,1}
\end{gathered}
$$

The average Nusselt and Sherwoood numbers, on the isothermal walls of the enclosure are defined as follows:

$$
\begin{aligned}
& \bar{N} u=\int_{00}^{11} \int_{0}^{1} N u \partial y \partial z \\
& \overline{S h}=\int_{00}^{11} \int h \partial y \partial z
\end{aligned}
$$

For each time step, the following convergence criterionissatisfied: 


$$
\sum_{1,2,3} \frac{\max \left|\psi^{n}-\psi^{n+1}\right|}{\max \left|\psi^{n}\right|}+\max \left|T^{n}-T^{n+1}\right|+\max \left|C^{n}-C^{n+1}\right| \leq 10^{-5}
$$

\section{Boundary conditions}

The current numerical simulations employ the following boundary conditions:

Temperature and concentration:

$$
\begin{gathered}
T(0 . y \cdot z)=C(0 . y \cdot z)=0 \text { For } x=0 \\
T(1 \cdot y \cdot z)=C(1 \cdot y \cdot z)=1 \text { For } x=1 \\
\left.\left.\frac{\partial T}{\partial y}\right)_{y=0 \text { and } 1}=\frac{\partial C}{\partial y}\right)_{y=0 \text { and } 1}=0 \\
\left.\left.\frac{\partial T}{\partial z}\right)_{z=0 \text { and } 1}=\frac{\partial C}{\partial z}\right)_{z=0 \text { and } 1}=0
\end{gathered}
$$

Vorticity:

$$
\begin{aligned}
& \text { At } \mathrm{x}=0 \text { and } \mathrm{x}=1: \omega_{1}=0, \omega_{2}=-\frac{\partial \mathbf{U}_{3}}{\partial \mathrm{x}}, \omega_{3}=\frac{\partial \mathbf{U}_{2}}{\partial \mathrm{x}} \\
& \text { At } \mathrm{y}=0 \text { and } \mathrm{y}=1: \omega_{1}=\frac{\partial \mathbf{U}_{3}}{\partial \mathrm{y}}, \omega_{2}=0, \omega_{3}=-\frac{\partial \mathbf{U}_{1}}{\partial \mathrm{y}} \\
& \text { At } \mathrm{z}=0 \text { and } \mathrm{z}=1: \omega_{1}=-\frac{\partial \mathbf{U}_{2}}{\partial \mathrm{z}}, \omega_{2}=\frac{\partial \mathbf{U}_{1}}{\partial \mathrm{z}}, \omega_{3}=0
\end{aligned}
$$

Vector potential:

$$
\begin{aligned}
& \text { At } \mathrm{x}=0 \text { and } \mathrm{x}=1: \frac{\partial \psi_{1}}{\partial x}=\psi_{2}=\psi_{3}=0 \\
& \text { At } \mathrm{y}=0 \text { and } \mathrm{y}=1: \psi_{\psi_{1}}=\frac{\partial \psi_{2}}{\partial y}=\psi_{3}=0 \\
& \text { At } \mathrm{z}=0 \text { and } \mathrm{z}=1:{ }_{\psi_{1}}=\psi_{2}=\frac{\partial \psi_{3}}{\partial z}=0
\end{aligned}
$$

Velocity on all walls: 


$$
U_{1}=U_{2}=U_{3}=0
$$

Simulations are accomplished for strong concentrated suspensions (Guram and Smith [48]). It characterized the case in which the microelements in the vicinity of the wall surface are incapable to rotate. Thus, the boundary conditions for micro-rotation vector are given as follows:

$$
\text { At } \mathrm{x}=0,1 ; \mathrm{y}=0,1 \text { and } \mathrm{z}=0,1: \vec{H}=0
$$

\section{Numerical procedure and code validation}

The FORTRAN language was used to write the numerical program and solve the mathematical model described above. The governing equations ((7)-(11)) were discretized using the finite volume method based on the central-difference scheme andsolved using the successive relaxation iteration scheme. Thegrids are uniform in all directions, and additional nodes are added onboundaries. A time-step of $10^{-4}$ and spatial mesh of $51 \times 51 \times 51$ was used during the numerical simulations and the convergence criterion given in equation (16) was satisfied. We used an Intel core i5 microprocessor for the numerical simulation, the time of execution was about7 $\mathrm{h}$ for $\mathrm{Ra}=10^{5}$. Hence, the $(51 \times 51 \times 51)$ grid was estimated to be appropriate for the present study at least for $\mathrm{Ra} \leq 10^{5}$ since it permits a good compromise between the computational cost (a significant reduction of the execution time) and the accuracy of the obtained results, as shown above in Table 2.

The finite volume method consists in defining mesh nodes inside the computation domain and in constructing a control volume around each node. The space is divided into a finite number of elementary volumes. The main nodes are located in the center of each volume and additional nodes on the boundaries of the domain. Figure 1(b) displays the mesh configuration of the present physical domain.

A grid independence study has been first carried out such that the exact number of grids for the present simulations is wisely determined. The grid independency analysis is presented in Table 2. The values of average Nusselt and Sherwood numbers on the hot wall at different grid sizes are calculated for $K=1, R a=10^{5}$ and $\varphi=0.00055$. A $51 \times 51 \times 51$ uniform grid is found to meet the requirements of both the grid independency study and the computational time limits. The percentages quoted in Table 2 represent relative errors corresponding to the above quoted value. 
Table 2Grid independence study ( $K=1, R a=10^{5}$ and $\left.\varphi=0.00055\right)$.

\begin{tabular}{clllll}
\hline Mesh size & $31 \times 31 \times 31$ & $41 \times 41 \times 41$ & $51 \times 51 \times 51$ & $61 \times 61 \times 61$ & $71 \times 71 \times 71$ \\
\hline$\overline{N u}$ & 3.0866 & 3.1165 & 3.1168 & 3.1025 & 3.0998 \\
& $(0.9689 \%)$ & $(0.0096 \%)$ & & $(0.45 \%)$ & $(0.54 \%)$ \\
\hline$\overline{S h}$ & 3.4044 & 3.4456 & 3.4454 & 3.435 & 3.4247 \\
& $(1.305 \%)$ & $(0.0017 \%)$ & & $(0.30 \%)$ & $(0.60 \%)$
\end{tabular}

Then, the present simulation is validated with the three-dimensional results of the works performed by Sezai and Mohamad [49]. The numerical results presented in this study are limited to water-based solutions. The comparisons are presented in Table 3. It can be concluded that there is agood agreement between the value of $\overline{N u}$ and $\overline{S h}$ of the present work and the selected one for water based solution.

Table 3Comparison with the results of Sezai and Mohamad [49] for $\operatorname{Ra}=10^{5}, \mathrm{Le}=10, \mathrm{Pr}=$ 10

\begin{tabular}{llll}
\hline$N$ & Authors & $\overline{N u}$ & $\overline{S h}$ \\
\hline 0 & Present work & 4.522 & 11.16 \\
& Sezai and Mohammad [49] & 4.532 & 11.046 \\
& Percentage difference & $0.22 \%$ & $1.032 \%$ \\
\hline-0.2 & Present work & 4.483 & 10.757 \\
& Sezai and Mohammad [49] & 4.473 & 10.736 \\
& Percentage difference & $0.22 \%$ & $1.20 \%$ \\
\hline-0.6 & Present work & 3.723 & 5.9572 \\
& Sezai and Mohammad [49] & 3.703 & 5.982 \\
& Percentage difference & $0.54 \%$ & $0.41 \%$ \\
\hline-1.8 & Present work & 1.406 & 6.772 \\
& Sezai and Mohammad [49] & 1.396 & 6.782 \\
& Percentage difference & $0.71 \%$ & $0.14 \%$ \\
\hline-2 & Present work & 1.367 & 7.204 \\
& Sezai and Mohammad [49] & 1.347 & 7.224 \\
& Percentage difference & $1.48 \%$ & $0.27 \%$ \\
\hline & & & \\
& & &
\end{tabular}

The validation of our three-dimensional numerical code of natural convection of nanofluid was also performed against the results generated by Kolsi et al. [50] for water- 
$\mathrm{Al}_{2} \mathrm{O}_{3}$ nanofluid as shown in Fig. 3. It can be seen from this figure that the solution of the present numerical code is in excellent agreement with the numerical results from Kolsi et al. $[50]$.

Afterwards, the proposed three-dimensional nanofluid model that takes into account the nanoparticles microrotation is validated against experimental data of Putra et al. [1]. To that end, a two-dimensional version of the present code is derived and the results are compared to those of Putra et al. [1] for natural convection of $\mathrm{Al}_{2} \mathrm{O}_{3} /$ water nanofluid.

Figure 4illustrates that the experimental data and our numerical results reveal similar trends. We realized a bi-dimensional natural convection of $\mathrm{Al}_{2} \mathrm{O}_{3}$ /water nanofluid simulations. One can cite, for example for $R a=4.5 \times 10^{6}$, the difference between our numerical results and experimental results of Putra et al. [1] is about $22.8 \%$ at $K=1$ then it is decreased to $2.26 \%$ at $K=3$. It can be clearly observed that the maximum difference between experimental study and our results for $K=3$ does not exceed $3.89 \%$ for all the Rayleigh numbers considered. Evidently, as the microrotation parameter $K$ increases our numerical results tend to the experimental data. Therefore, this evidences the role of the nanoparticles microrotation and the need to consider its influence in the proposed simulations.

As a summary, in all validations, a reasonable agreement can be established between the present investigation results and those from earlier published works $[1,49,50]$.

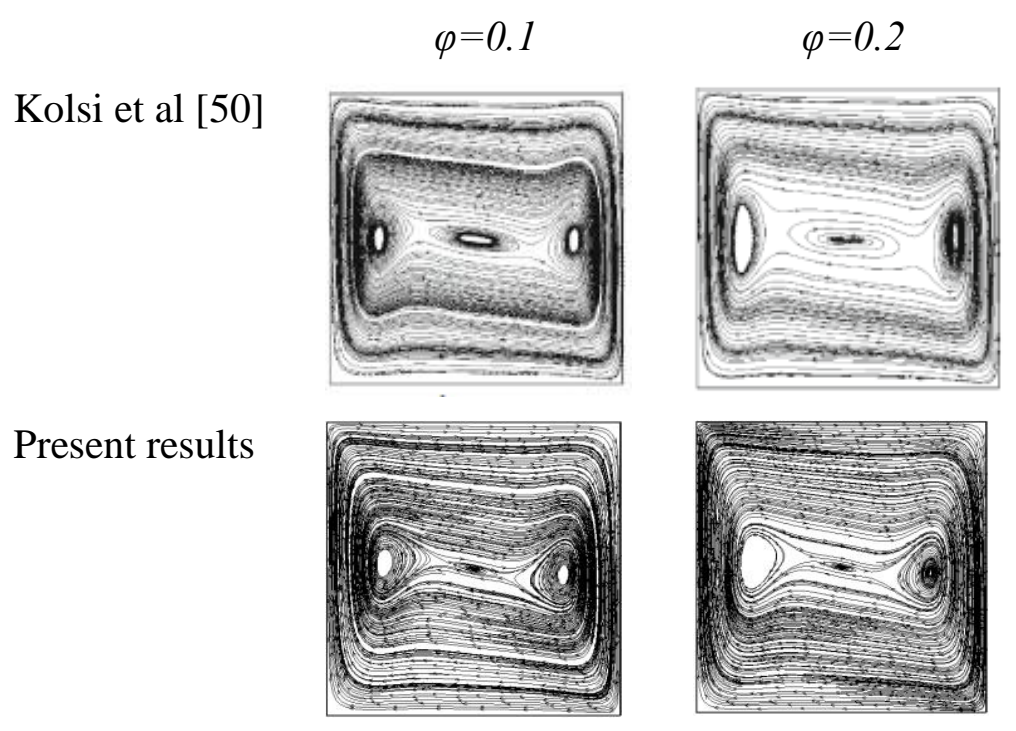

Fig. 3 Comparison of the streamlines with the results of Kolsi et al. [50] ( $P r=6.2$, and

$$
\left.R a=10^{6}\right)
$$




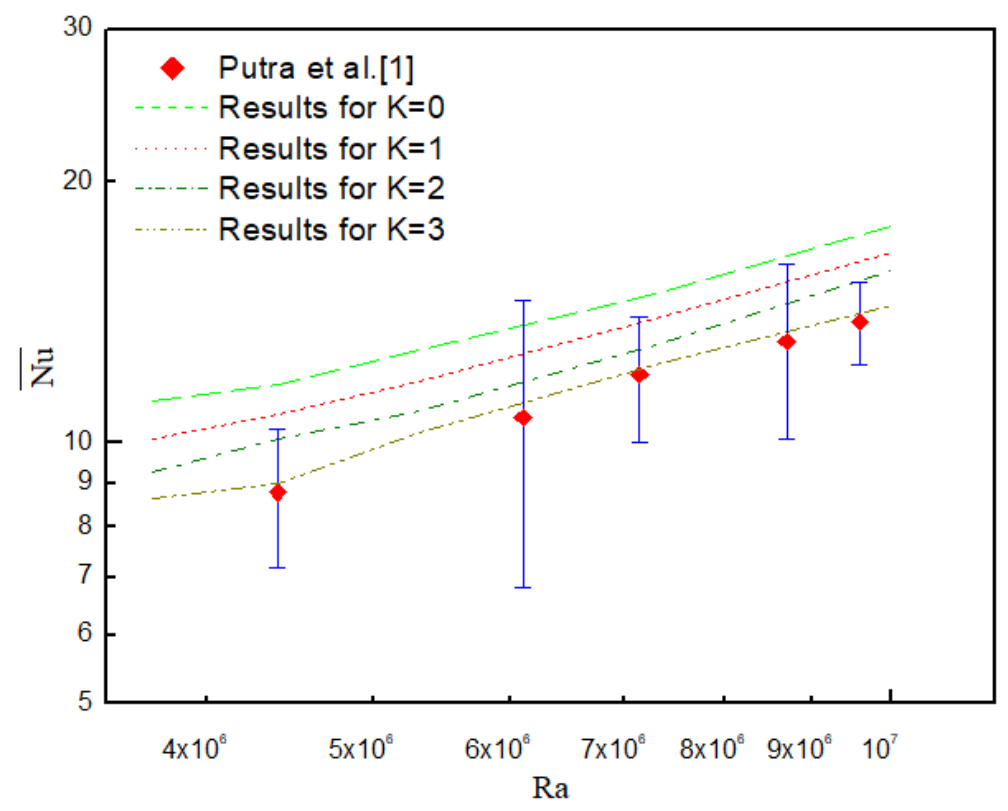

Fig. 4 Comparison of the average Nusselt number with experimental results of Putra et al. [1] versus Rayleigh number with increasing vortex viscosity parameter $K$ for $\varphi=4 \%$

\section{Results and discussion}

The thermo-solutal natural convection in a cubic cavity filled with a multi-walled CNT/water micropolar nanofluid stabilized with two types of surfactants is computationally investigated. Water solution was considered as the base fluid for all simulations. The Prandtl and Lewis numbers are fixed respectively equal to $\operatorname{Pr}=7$ and $L e=1$ (Aly and Raizah [14]). The simulations were carried out for the range of Rayleigh numbers $\left(10^{4} \leq R a \leq 10^{6}\right)$, micropolar parameter $(0 \leq K \leq 10)$, buoyancy ratio $(-1 \leq N \leq 0)$, and nanoparticle volume fractions $(0.055 \% \leq \varphi \leq 0.557 \%)$, to investigate the effects of various micropolar nanofluid on flow dynamics, heat and mass transfer characteristics. The effect of types of surfactants used to the stability of nanofluid has been investigated. Moreover, the influence of micropolar /nonmicropolar model is analyzed by analyzing streamlines, isotherms, isoconcentrations and average Nusselt and Sherwood numbers.

Figure 5 displays, projections of flow lines in the main plane $(\mathrm{z}=0.5)$ for various nanoparticles volumes fractions for micropolar/non-micropolar nanofluid stabilized with Lignin (N2) and sodium polycarboxylate (N3). In the two cases, the main flow direction is clockwise in the x-y plane. Also, it can be mentioned that the intensity of the flow depends on the volume fraction of the nanoparticles. Indeed, for $\varphi \leq 0.055 \%$ the intensity increases while 
for $\varphi>0.055 \%$ the intensity decreases for the case N2. While the critical value of the volume fraction for the $\mathrm{N} 3$ case is $0.111 \%$. This profile obtained from the flowlinesis due to the improved thermal properties of the nanofluid for a small volume fraction of the nanoparticles, whereas the increase of nanoparticles concentration results in an enhancement of dynamic viscosity which implies a reduction in the intensity of the flow. It can be noted also that irrespective of the value of nanoparticles's volume fraction the N2 nanofluid has a higher flow intensity compared to N3. It is due to the fact that $\mathrm{N} 2$ has a lower dynamic viscosity. Moreover, it can be observed in this figure that when considering the micropolar theory, the principal flow strength ismore decelerated for both two types of surfactants. This is because of an enhancement in the vortex viscosity that results in the total viscosity increase of the fluid flow, thus decelerating the fluid flow.

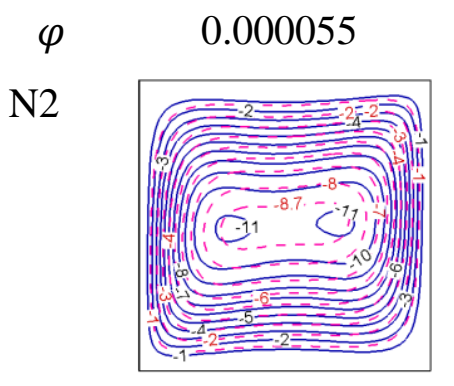

0.00055

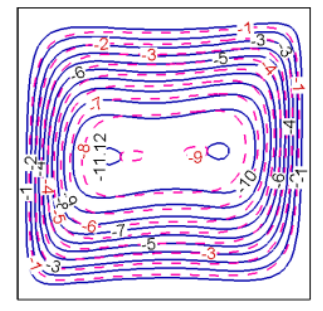

N3
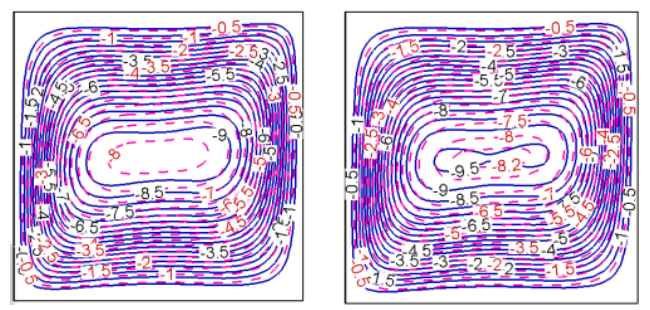

0.00111
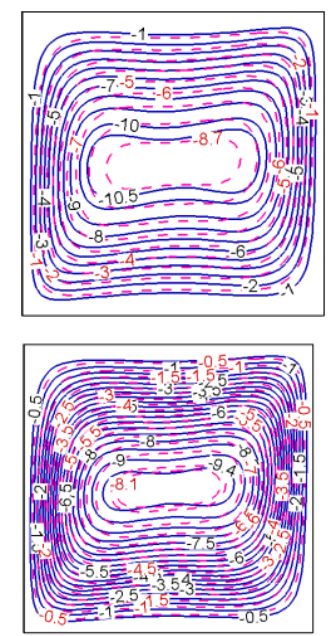

0.00557
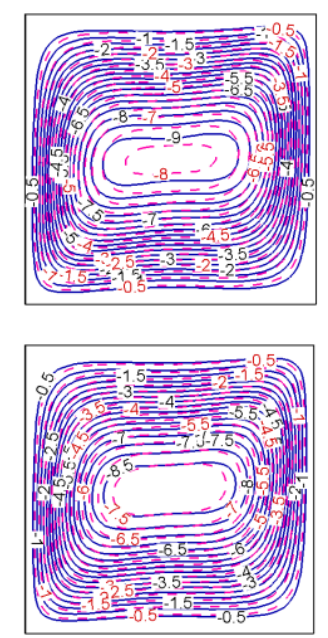

Fig.5 Projections of flow lines, in the main plane $\mathrm{z}=0.5$ for $\mathrm{N} 2 / \mathrm{N} 3$ micropolar nanofluid (dashed lines) and non-micropolar nanofluid (solid lines) for different nanoparticles's volume fraction, for $R a=10^{5}$ and $N=-0.2$.

For more clarification, Fig.6 elucidates the variations of the horizontal and vertical velocity components for $\mathrm{z}=0.5$ and $R a=10^{5}$ obtained by usingN2/N3 nanofluid with the micropolar model to evidence the consequence of nanoparticles concentration increase. Based on this figure, the amplitudes of the velocities $U_{1(0.5, y 0.5)}$ and $U_{2(x, 0.5,0.5)}$ are ameliorated when $\varphi$ is less than the critical value and then are deteriorated when it exceeds this value. Similarly, it can also be seen in Fig.6 that when the N2 nanofluid is employed, the intensity of the principal flow is higher than for N3 nanofluid. This can be explained by the lower dynamic viscosity of $\mathrm{N} 2$ compared to $\mathrm{N} 3$. 

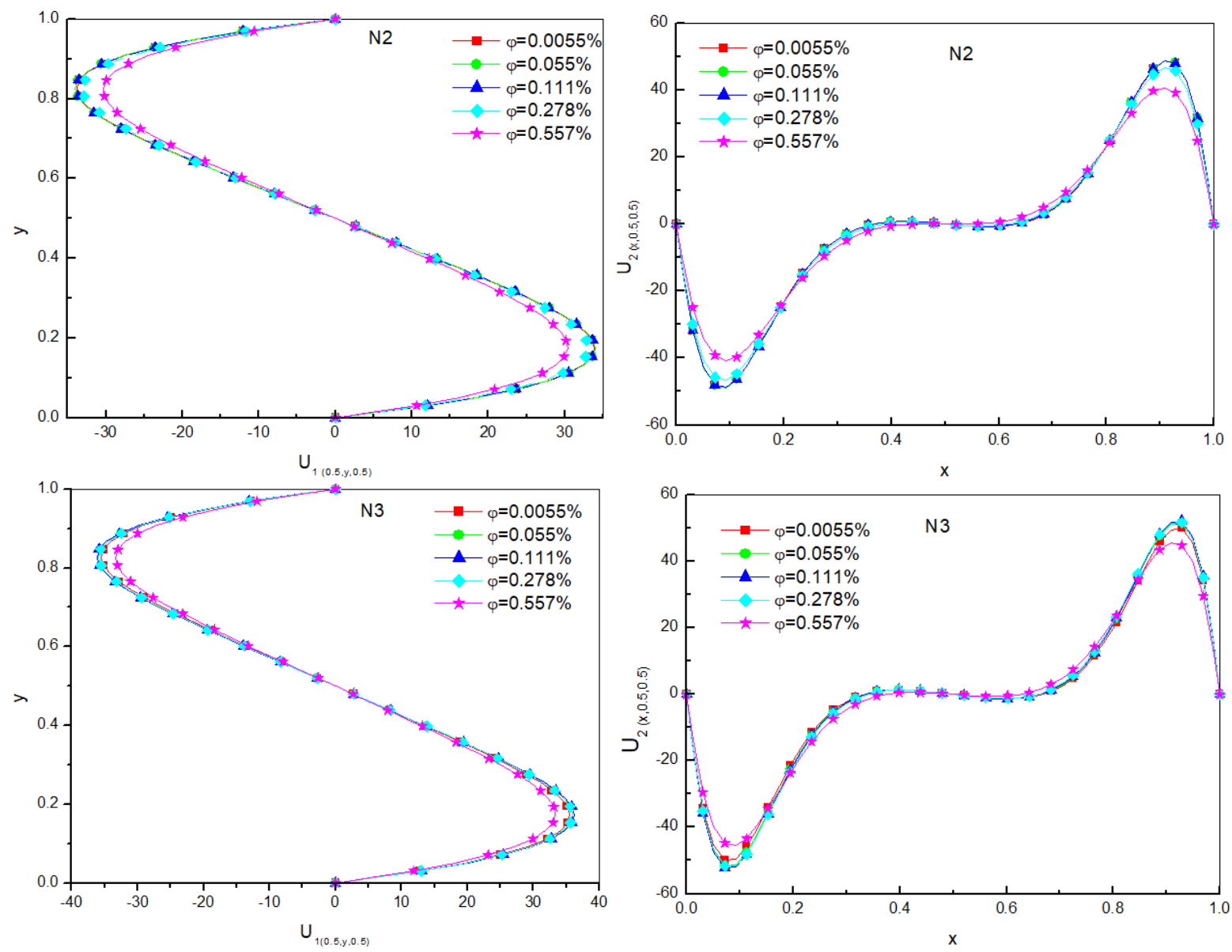

Fig. 6 Variation of components of velocity $U_{1(0.5, y, 0.5)}$ and $U_{2(x, 0.5,0.5)}$ versus different nanoparticles volume fraction, for $\mathrm{N} 2$ and $\mathrm{N} 3$ nanofluid, for $R a=10^{5}, K=1$ and $N=-0.2$.

Figure 7 shows the variations of maximum transverse velocity with the nanoparticle's volume fraction for micropolar/non-micropolar nanofluid stabilized with Lignin (N2) and sodium polycarboxylate (N3). Thus, the effects of nanoparticles' volume fraction on the three-dimensional flow are explored. This figure shows, irrespective of the model used,that the maximum transverse velocity is improved by increasing $\varphi$ to the critical value then the velocity tends to decrease. Also, it is clearly noted that N2 have higher transverse velocity compared to N3. For example, for $\varphi=0.0055 \%$ it exists a $27.63 \%$ difference between the two cases. In addition, it isinteresting to note that the non-miropolar nanofluid $(K=0)$ has the highest maximum transverse velocity compared to the micropolar nanofluids $(K \neq 0)$ for all the considered values of nanoparticles volume fraction. 

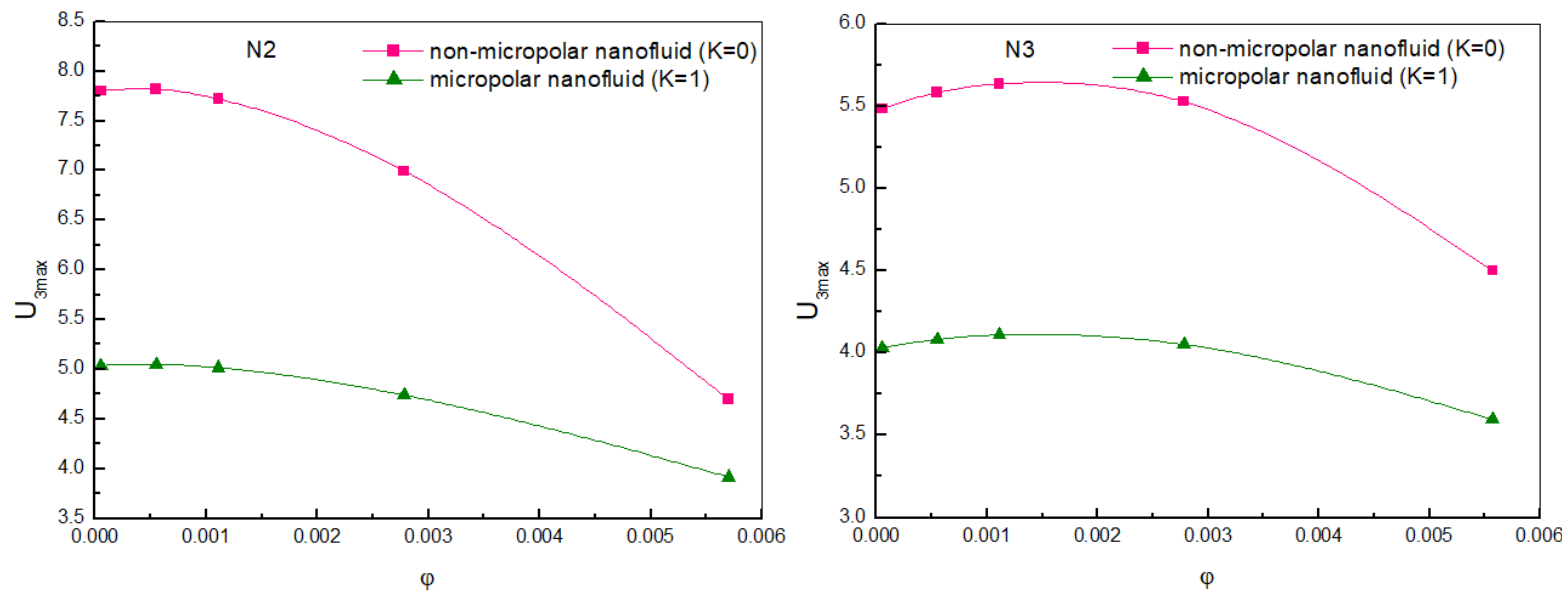

Fig. 7 Variation of maximum of transversal velocity $U_{3 \max }$ according to the nanoparticles volume fraction for $\mathrm{N} 2$ and $\mathrm{N} 3$ micropolar/non-micropolar nanofluid for $R a=10^{5}$ and $N=-0.2$.

Figure 8 shows the variations of average Nusselt number $\overline{N u}$ and Sherwood number $\overline{S h}$ with nanoparticles' volume fraction for micropolar/non-micropolar nanofluid stabilized with Lignin (N2) and sodium polycarboxylate (N3). Irrespective to the considred type of nanofluids, $\overline{N u}$ and $\overline{S h}$ are lower when the micropolar nanofluid model is under consideration, and are increased when the non-micropolar fluid model is under consideration irrespective of the values of nanoparticles volume fraction. It is noticed that for the two types of surfactants used, the average Nusselt and Sherwood numbers increase in the area where the value of the volume fraction of nanoparticles is less than its critical value. This is because the thermal performance of water solution is improved by the addition of nanoparticles. Afterwards, the heat and mass transfer rates decrease in the area where the value of the nanoparticles volume fraction is greater than its critical value due to the predominant influence of viscosity over thermal properties. It can be seen also from Fig.8, for the case where the surfactant lignin is used, the average Nusselt and Sherwood numbers are greater than in the case of the surfactant sodium polycarboxylate is employed. 

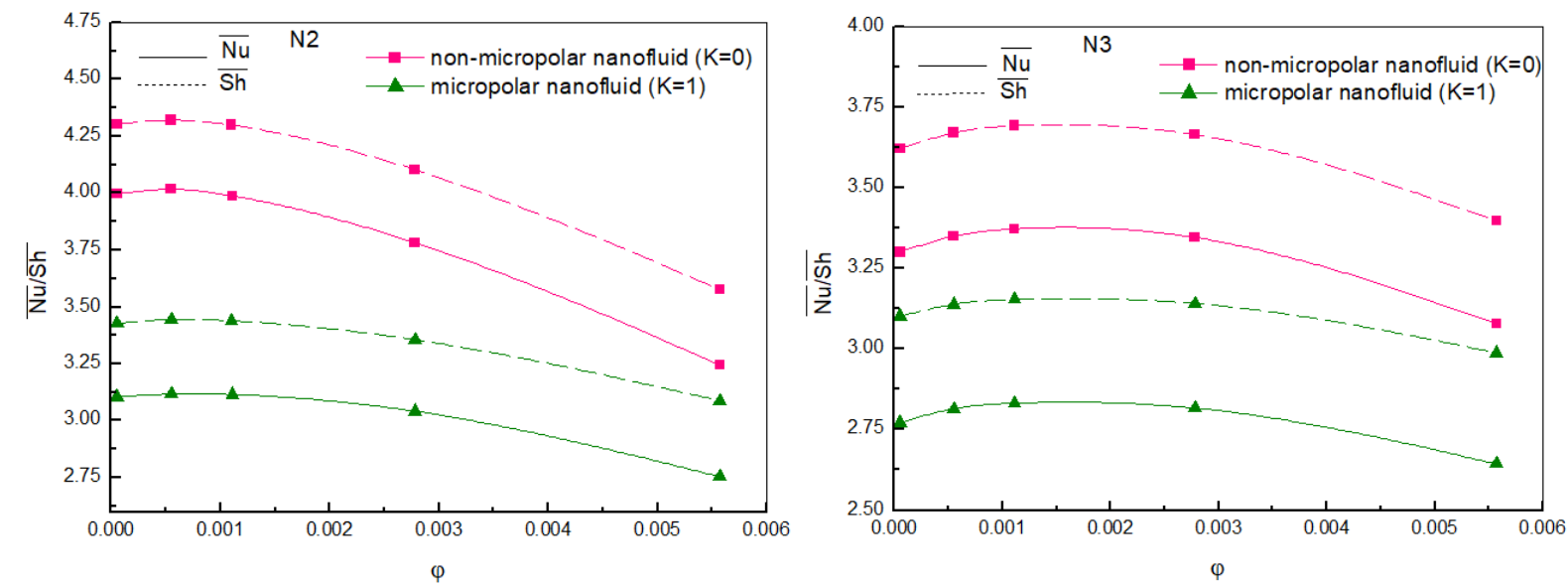

Fig. 8 Variation of $\overline{N u}$ and $\overline{S h}$ on the hot wallwithnanoparticles volume fraction for both micropolar and non-micropolar N2/N3 nanofluid models for $R a=10^{5}$ and $N=-0.2$.

The isocontours of the local Nusselt number determined on the hot wall of the cavity are shown in Fig. 9 for different volume fraction of nanoparticles for micropolar/non-micropolar nanofluid stabilized with Lignin (N2) and sodium polycarboxylate (N3). It is clearly noted that the heat transfer decreases from bottom to top. This figure shows that the local Nusselt number varies in the vertical direction (y). Furthermore, in the horizontal direction $(\mathrm{z})$ the isocontours of the local Nusselt number are quite uniform in the central area of the hot wall. Quantitatively, the local Nusselt maximum value decreases from non-micropolar nanofluid model to micropolar model. This proves the variation of the previous figure. It is also noted that the heat and the mass transfer increase with the enhancement of nanoparticles volume fraction until a critical value of $\varphi$. Beyond this value of $\varphi$, it decreases. 
$\varphi$

$\mathrm{N} 2$

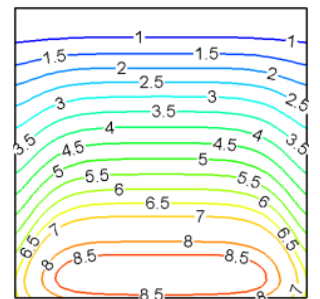

$\mathrm{N} 2$

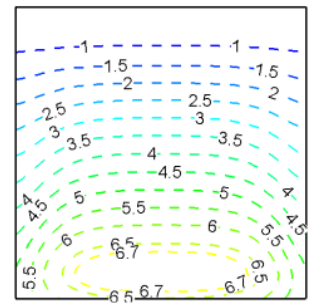

N3

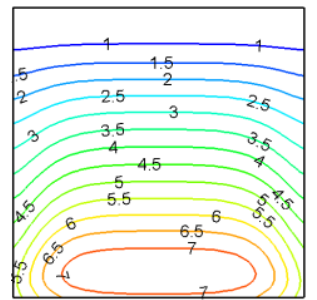

N3

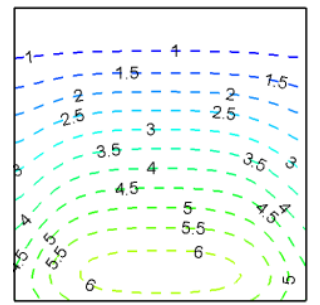

0.00055
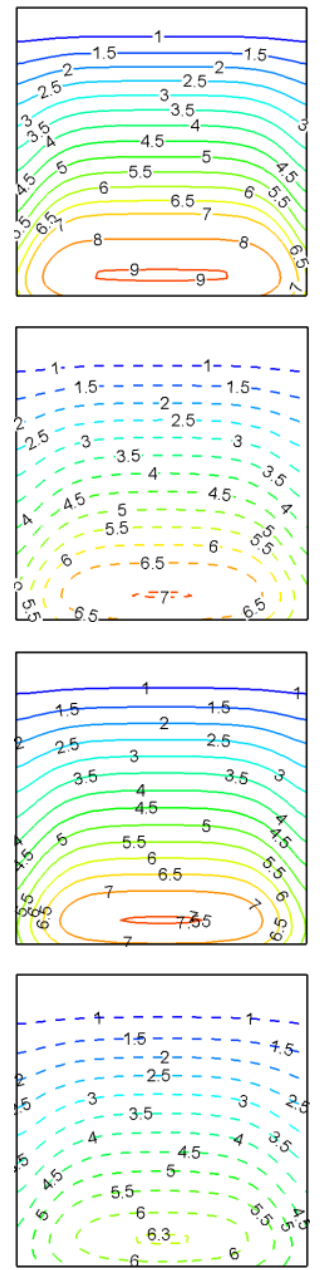

0.00111
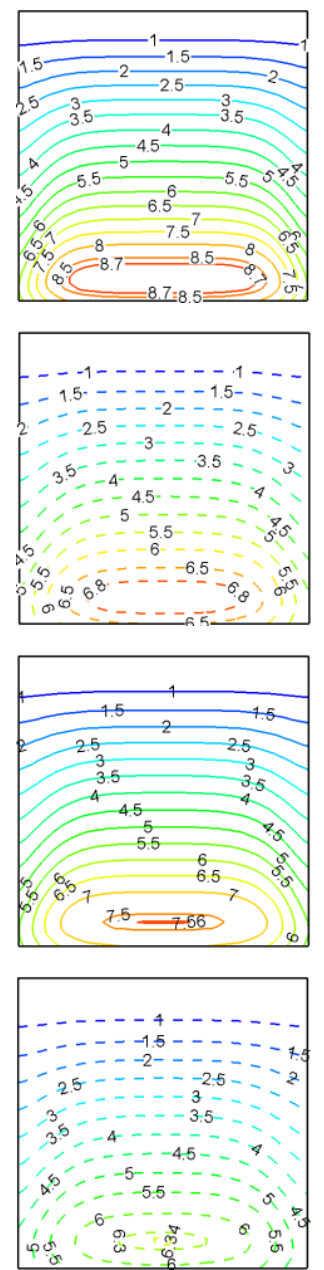

0.00557
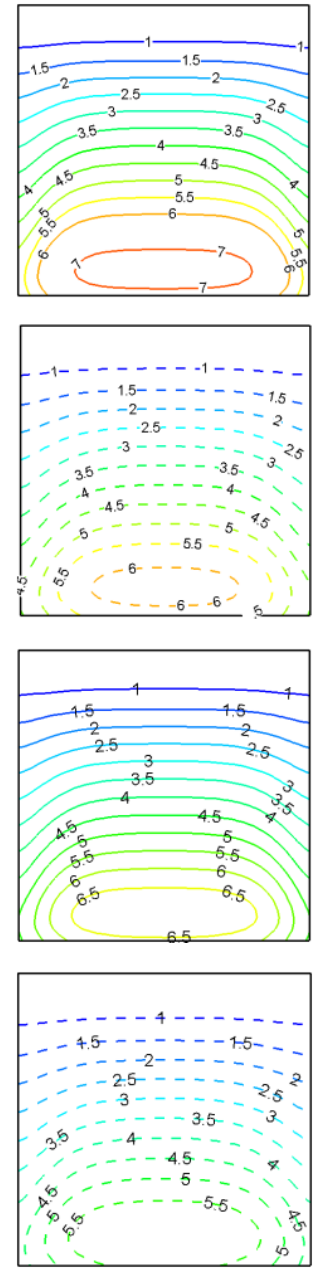

Fig.9 Distributions of local Nusselt number on the hot wall for some values of nanoparticles volume fraction for both $\mathrm{N} 2$ and N3 non-micropolar nanofluid (solid lines) and micropolar nanofluid (dashed lines) for $R a=10^{5}$ and $N=-0.2$.

Figures 10 shows the distribution of flow lines on the mid $x-y$ plane in addition to the isotherms and the isoconcentrations, for $\mathrm{N} 2$ micropolar nanofluid obtained for different buoyancy fraction.

At therather low buoyancy ratio, the flow is mainly dominated by the thermal buoyancy force, so that the main flow direction is clockwise in the $x-y$ plane. As the buoyancy ratio is increased to -1 , the isotherms and isoconcentrations have a horizontal direction and are gradually stacked near the active walls. The vertical thermal and solutal stratifications at the centre of the cavity are eliminated and the isotherms and isoconcentrations become straight up as $\mathrm{N}$ reached -1 . They are quasi-equidistant except near the active walls. The intensity of the flow becomes weaker and the flow pattern changes from a structure with two inner cells to a cell with only one vortex. It is worthy to note that the projection of flow lines on the mid X-Y 
plane are notclosed, but have a spiral form. The spiralling of the streamlines is an indication of the three-dimensional character of the flow field for which the fluid particles move from one constant $\mathrm{Z}$ plan to another.

At $\mathrm{N}=-1$, the effects of thermal and compositional re-circulations are of comparable magnitude.The resulting flow structure consists of one clockwiserotating main roll due to thermalbuoyancy force and twosmaller rolls due to an opposing solutal gradient at the topand the bottom, both rotating in the counter-clockwise direction.

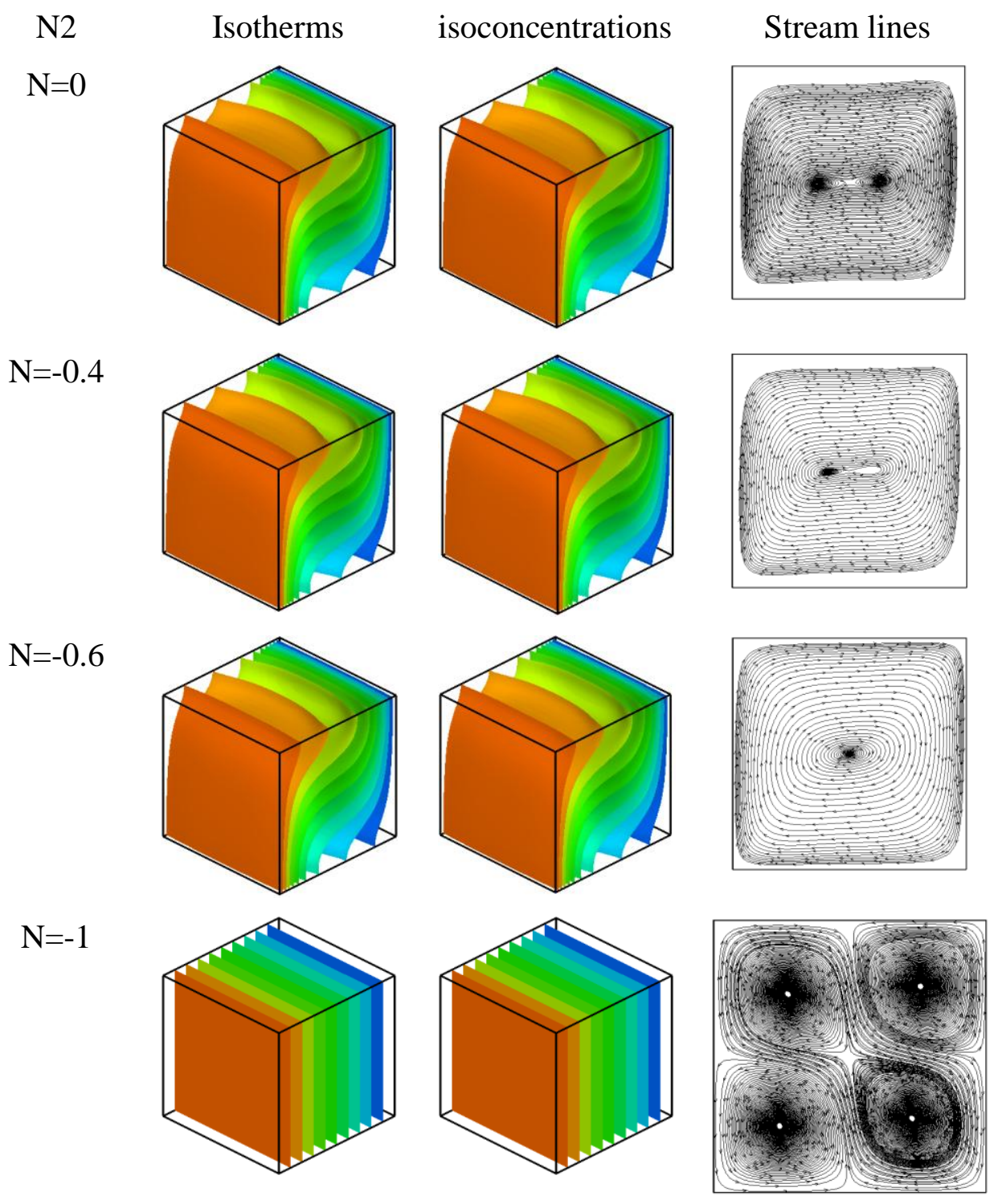

Fig. 10 Influence of the buoyancy ratio $\mathrm{N}$ on isotherms, isoconcentrations and the projections of flow lines in the main plane $\mathrm{z}=0.5$ for $\mathrm{N} 2, K=1, \mathrm{Ra}=10^{5}$ and $\varphi=0.00055$

Figure 11 illustrates the variations of the average Nusselt and Sherwood numbers calculated on the hot wall versus the buoyancy ratio $N$ for both micropolar nanofluid $(\mathrm{K}=1)$ and nonmicropolar nanofluid $(\mathrm{K}=0)$. 
It is understood from this figure that both $\overline{\mathrm{Nu}}$ and $\overline{\mathrm{Sh}}$ have a quasi-similar profile for both micropolar and non-micropolar N2/N3 nanofluid models. As the $\mathrm{N}$ is decreased in a negative direction until $\mathrm{N}=-1$, both the values of $\overline{\mathrm{Nu}}$ and $\overline{\mathrm{Sh}}$ show declining tendency.

The average heat and mass transfer rates are higher for $\mathrm{K}=0$ when compared to micropolar nanofluid $(K \neq 0)$ irrespective of the used surfactants. This phenomenon reflects the fact that increasing the value of vortex viscosity parameter results in an enhancement of the total viscosity in fluid flow and hence the heat and mass transfer rates are retarded.

It is alsoimportant to note that the comparison between figures of Lignin (N2) and sodium polycarboxylate (N3) reveals that employing a nanofluid stabilized with Lignin (N2) ameliorates the average Nusselt and Sherwood numbers more than that of sodium polycarboxylate (N3).
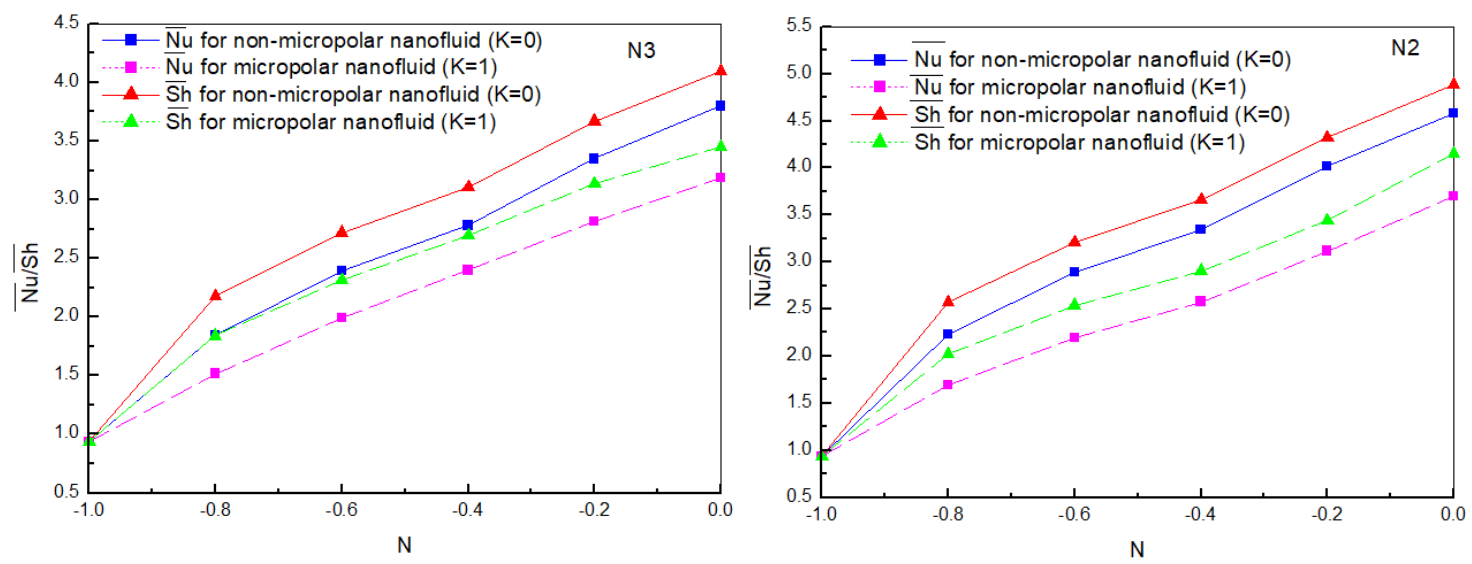

Fig. 11 Variation of the average Nusselt and Sherwood numbers according the buoyancy

$$
\text { fraction } N \text { for } \varphi=0.055 \% \text { and } R a=10^{5}
$$

Figure 12 illustrates the variations of the horizontal and vertical velocity components in the plane $(\mathrm{z}=0.5)$ with respect to the Rayleigh number for micropolar CNT/water nanofluid stabilized with Lignin (N2) and sodium polycarboxylate (N3). The flow regime is intensified and the magnitude of each velocity component is increased with the increase in the Rayleigh number. Moreover, the difference between two nanofluids is much greater for $R a \geq 10^{5}$. 

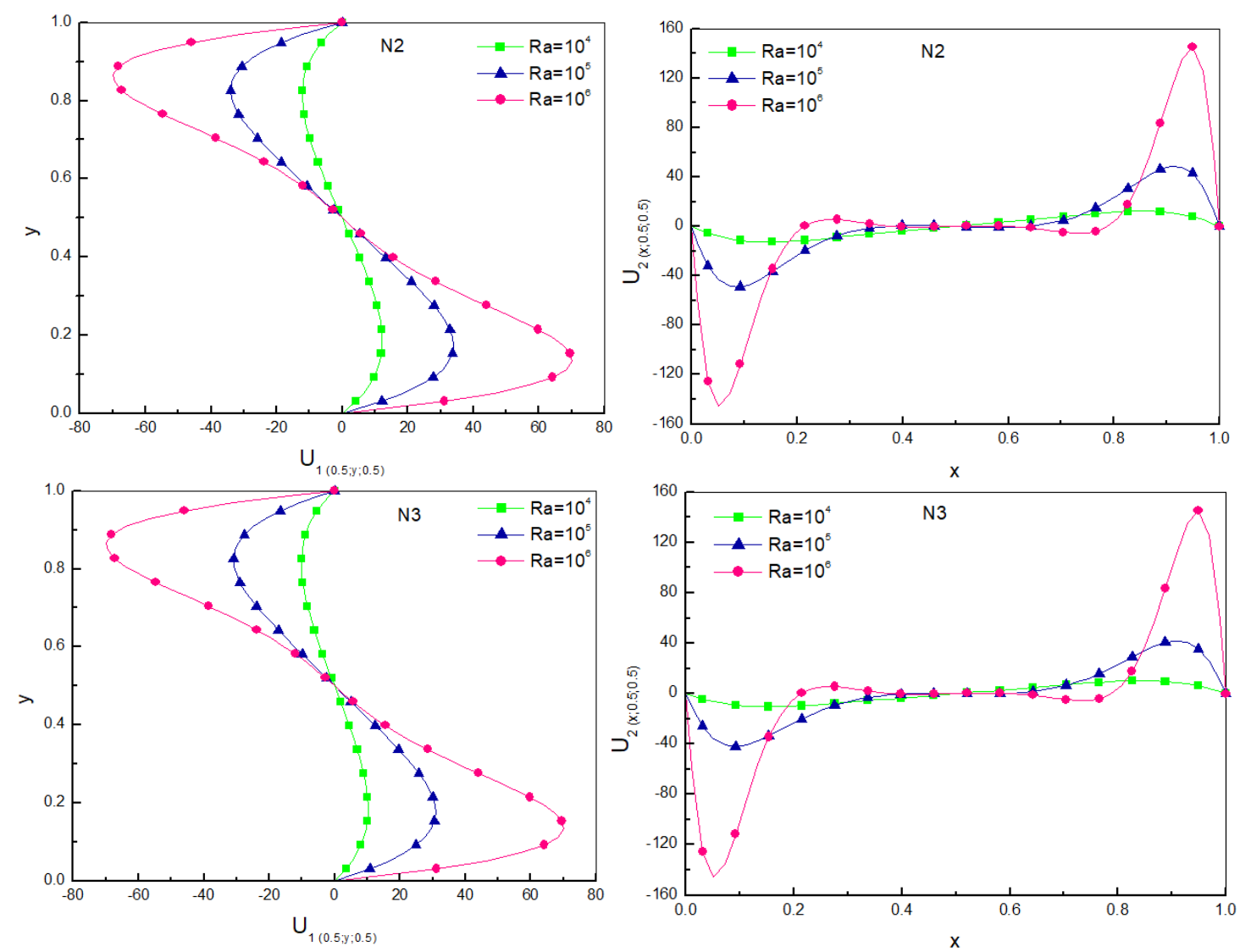

Fig. 12 Variations of the horizontal and vertical velocity components at $\mathrm{z}=0.5$ plane for $\mathrm{N} 2 / \mathrm{N} 3$ micropolar nanofluid, for different Rayleigh numbers, $K=1, N=-0.2$ and $\varphi=0.00055$

Figure 13 shows the variations of $\overline{N u}$ and $\overline{S h}$ with nanoparticles' volume fraction for different values of $R a$ and with micropolar CNT/water nanofluid stabilized with Lignin (N2) and sodium polycarboxylate (N3). Regardless of the nanoparticles' volume fraction, the heat and mass transfer increase with the increase in values of $R a$.It can be observed that the heat and mass transfer increase in the area where the value of the nanoparticles volume fraction is less than a critical value. This is because the thermal performance of water solution is improved by the addition of nanoparticles. Afterwards, the heat and mass transfer rates decrease in the area where the value of the nanoparticles volume fraction is greater than the critical value due to the predominant influence of viscosity over thermal properties. 

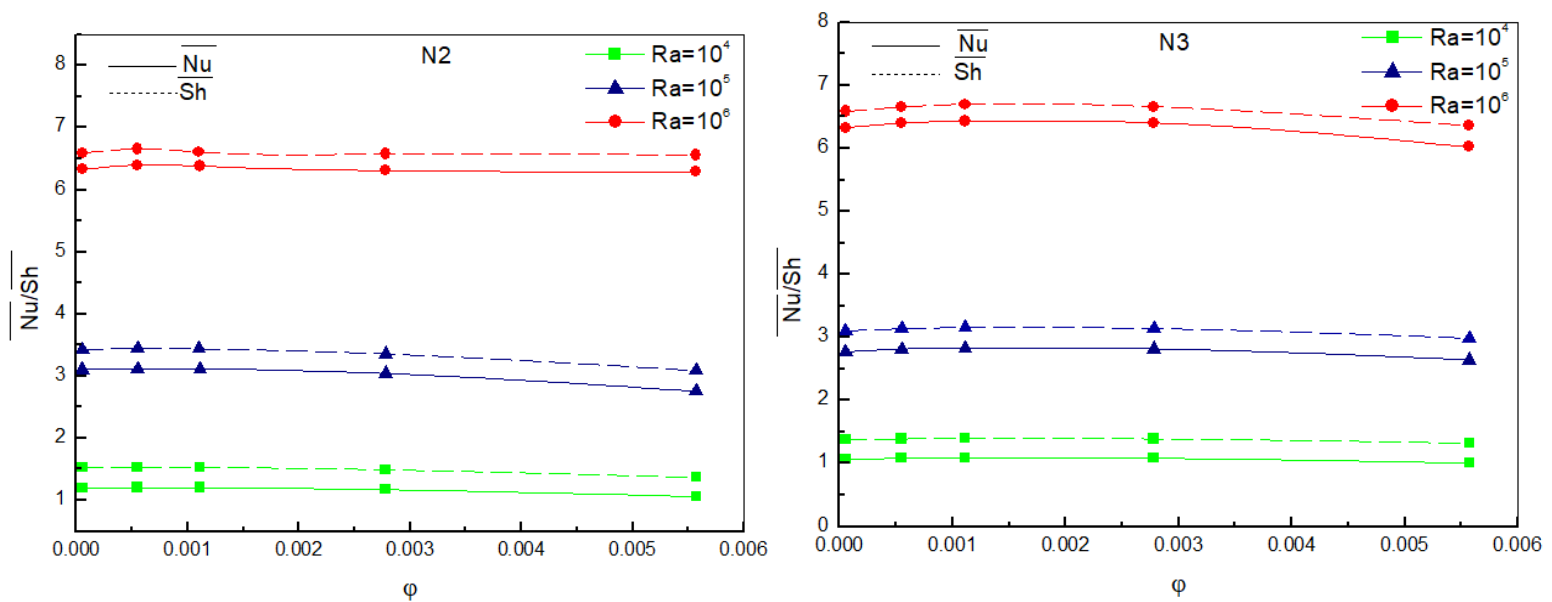

Fig. 13 Variation of the average Nusselt and Sherwood numbers on the hot wall according to the Rayleigh number $R a$ and nanoparticles'svolume fraction $\varphi$ for $K=1$ and $N=-0.2$

To study the effect of the Rayleigh number on the three-dimensional character of the flow, we present in Fig.14 the variations of the maximum transversal velocity $\mathrm{U}_{3 \max }$ according to the Rayleigh number $R a$ and for volume fraction ranging from 0.0055 to $0.557 \%$ for $\mathrm{N} 2$ and $\mathrm{N} 3$. This figure shows that for both types of surfactant, the maximum of transversal velocity increases by increasing $R a$. In addition, the three-dimensional character of the flow depends on nanoparticles volume fraction. In fact, it increases when $\varphi$ is less than a critical value, then $\mathrm{U}_{3 \max }$ decreases when $\varphi$ is higher than their critical value.
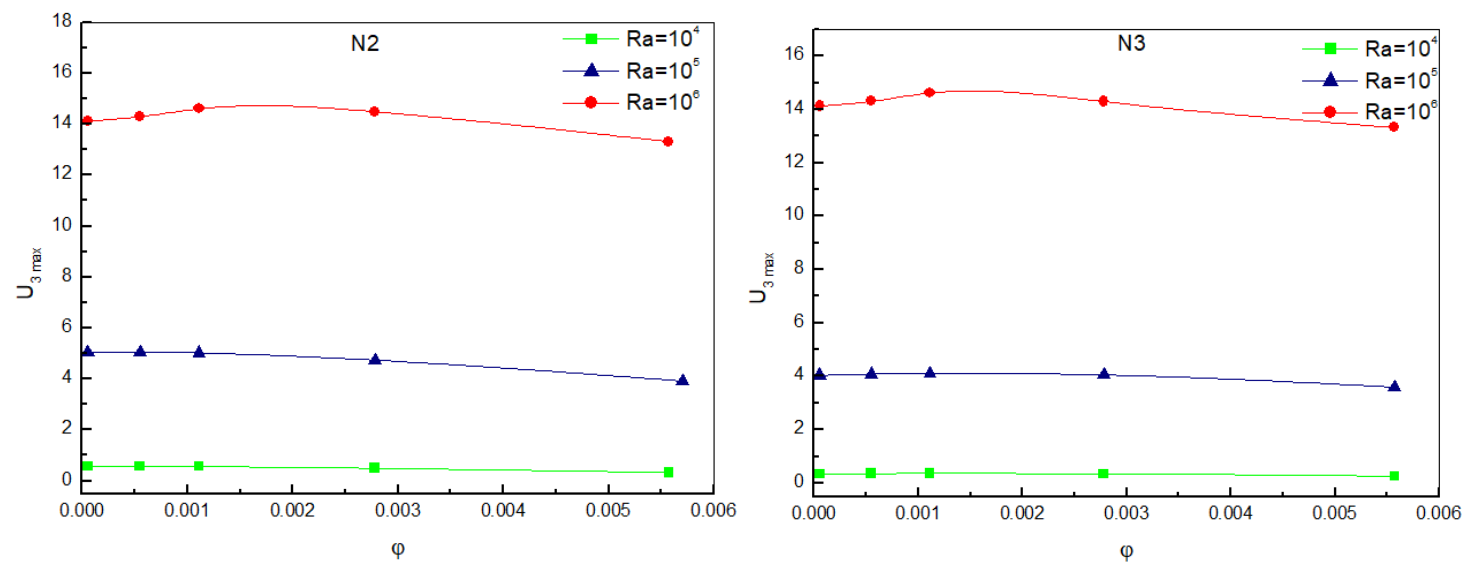

Fig.14 Variation of maximum of transversal velocity U3max versus the Rayleigh number Ra and nanoparicles volume fraction $\varphi$ for both $\mathrm{N} 2$ and N3 micropolar nanofluid for $K=1$ and

$$
N=-0.2 \text {. }
$$

In order to clearly show the three-dimensional character of the flow, Fig. 15 presents several particle trajectories for different Rayleigh numbers for micropolar CNT/water 
nanofluid stabilized with Lignin (N2) and sodium polycarboxylate (N3).As observed in Fig.15, the flow lines have a spiral form. This spiraling form is an indication of the threedimensional character of the flow field for which the fluid particles move from one constant $\mathrm{z}$ plane to another. This figure also shows that as the Rayleigh number $R a$ increases, the flow exhibits two central vortexes, mounting up along the cold wall and falling along the hot wall of the cavity.

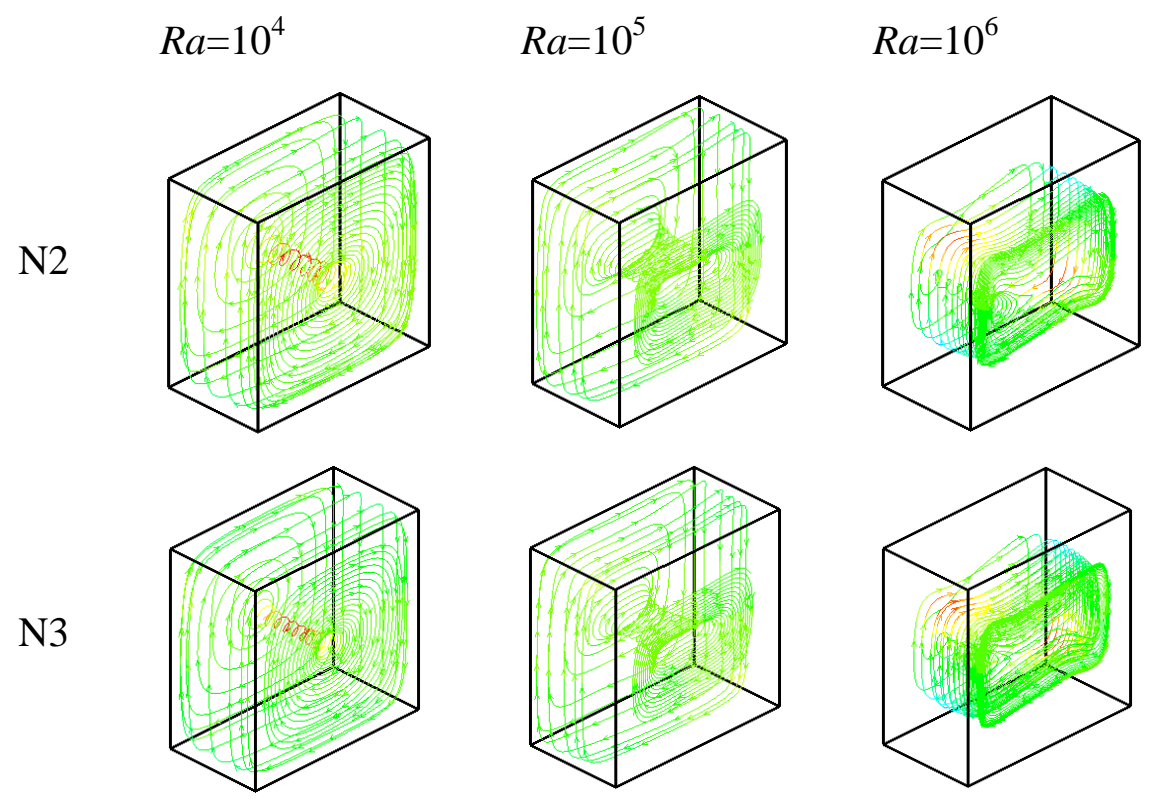

Fig.15: Some particles trajectories for different Rayleigh numbers for N2 and N3 micropolar nanofluidfor $\varphi=0.00055, N=-0.2$ and $K=1$

Figure16 shows the variation of the average Nusselt and Sherwood numbers with time $t$ for N2 and N3 for two Rayleigh numbers. It can be seen that the Nusselt and Sherwood numbers lines begin with a sudden sharp initial increase. After attaining certain values, these lines shift upward and pass through a transitional state. Finally, the average Nusselt and Sherwood numbers curves become stable. In addition, it can be concluded that, the time required to attain the steady state is shorter for higher Rayleigh numbers and longer for lower Rayleigh numbers. 

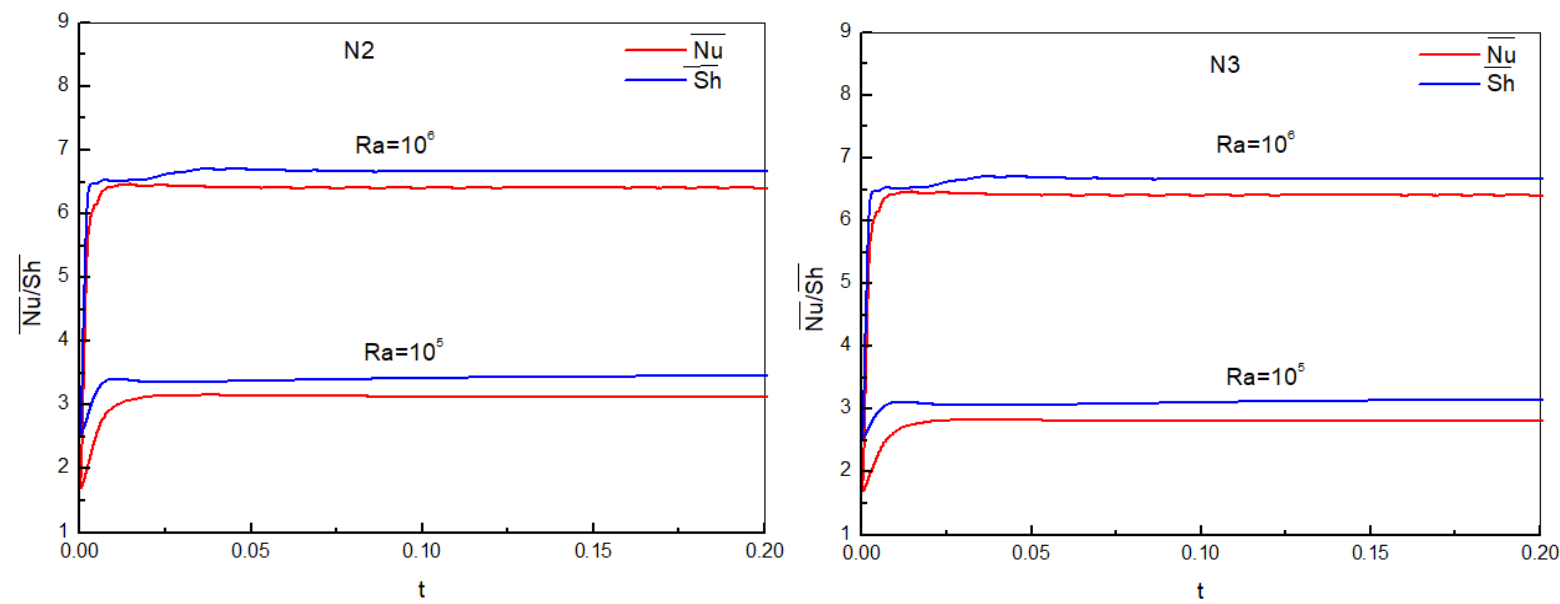

Fig. 16: Time-dependent Nusselt and Sherwood numbers for N2 and N3 micropolar nanofluid for $K=1, N=-0.2$ and $\varphi=0.055 \%$

Figures 17 and 18 show respectively the distribution of flow lines on the mid $\mathrm{x}-\mathrm{y}$ plane for $\mathrm{N} 2$ and $\mathrm{N} 3$ for $\varphi=0.055 \%$ and $\varphi=0.557 \%$ for different values micropolar parameter K. It is observed that when $\mathrm{K}$ increases the flow intensity decreases for fixed nanoparticles volume fraction. The physics behind the decrease of flow intensity can be explained by an increase in the micro-constituents' concentration that would lead to an amelioration in the vortex viscosity so then an increase in the total viscosity, inducing low velocity.

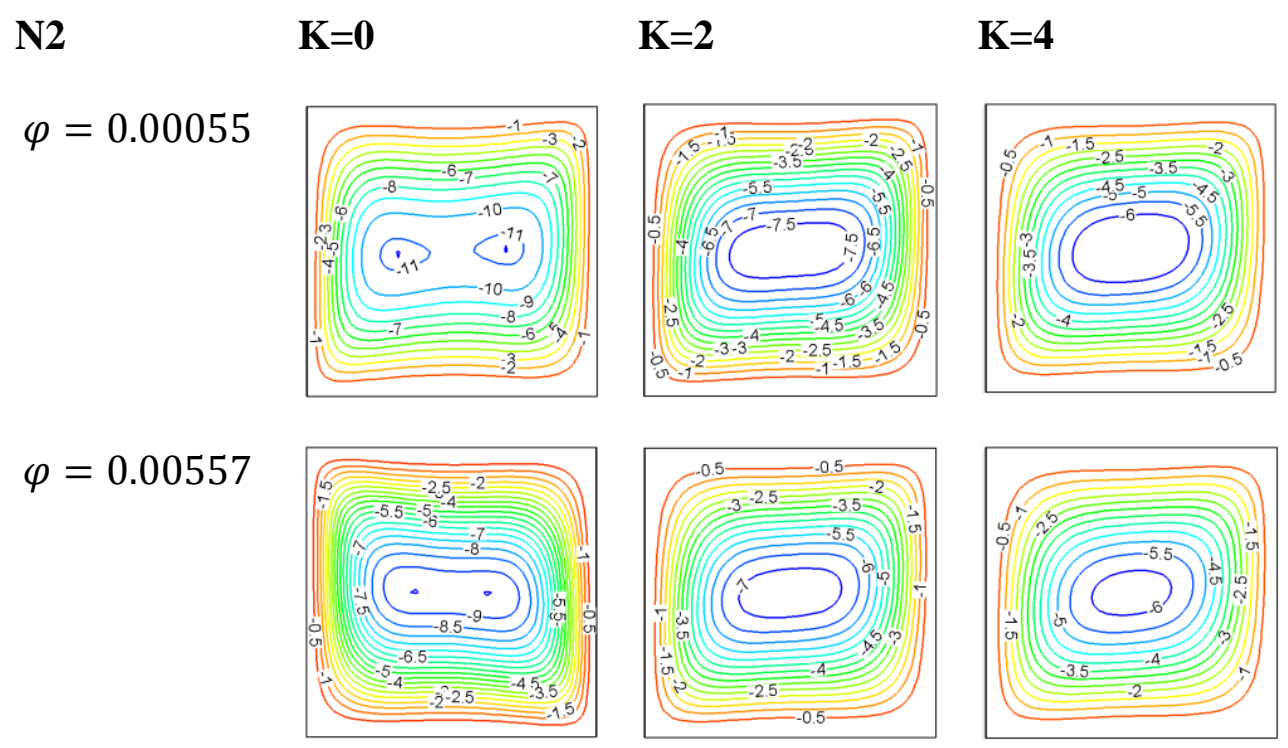

Fig.17 Projections of flow lines, in the main plane $\mathrm{z}=0.5$ for N2 micropolar nanofluid for different nanoparticles' volume fraction and different micropolar parameter, for $R a=10^{5}$ and $N=-0.2$. 
N3

$\varphi=0.00055$

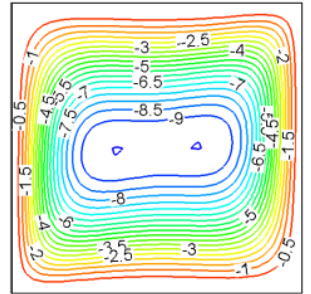

$\varphi=0.00557$

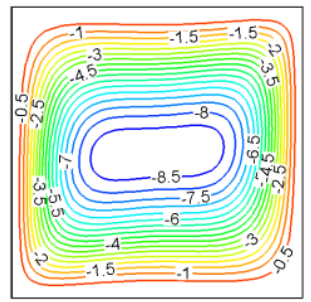

$\mathrm{K}=\mathbf{2}$
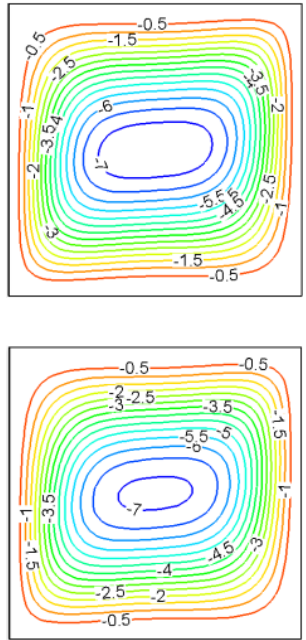

$K=4$
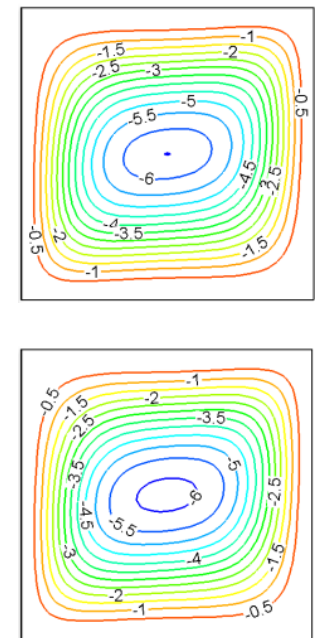

Fig.18 Projections of flow lines, in the main plane $\mathrm{z}=0.5$ for N3 micropolar nanofluid for different nanoparticles' volume fraction and different micropolar parameter, for $R a=10^{5}$ and $N=-0.2$.

Figure 19 depicts the variation of the average Nusselt and Sherwood numbers as functionof the vortex viscosity parameter $K$ for two values of nanoparticles volume fraction. The average heat transfer rates are greater for non-micropolar nanofluid $(K=0)$ when compared to micropolar nanofluid $(\mathrm{K} \neq 0)$. Figure 19 explains also that the values of $\overline{N u}$ and $\overline{S h}$ are decreased with an increase in $\mathrm{K}$ for both micropolar CNT/water nanofluid stabilized with Lignin (N2) and sodium polycarboxylate (N3). The physicsbehind the decrease in values of $\overline{N u}$ and $\overline{S h}$ can be explained as follows: an enhancing in the micro-constituents' concentration would lead to an amelioration in the vortex viscosity and the major part of the energy of the system is consumed for developing the gyrational velocities. As a result, the natural movement of molecules in the fluid was limitedand compensated by a decrease in heat and mass transfer rates. 

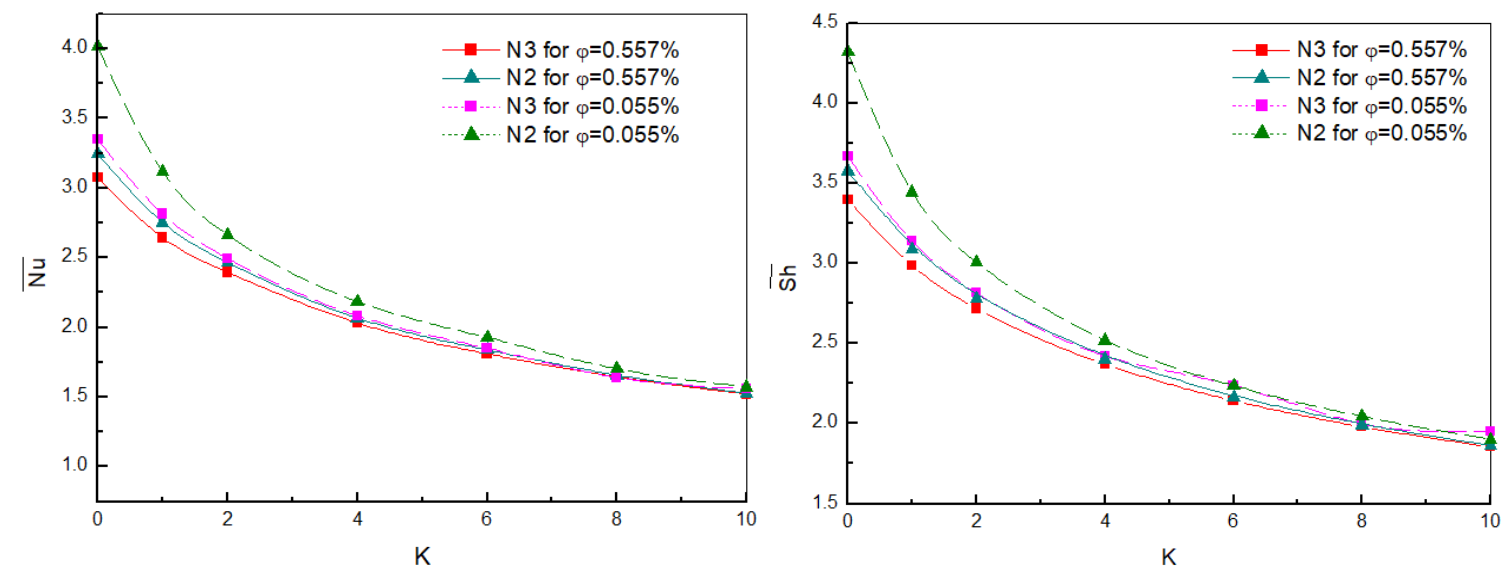

Fig. 19 Variation of average Nusselt and Sherwood numbers according the micropolar vortex parameter $K$ for $\varphi=0.055 \%, \varphi=0.557 \%$ and $N=-0.2$

Figure20 illustrates the variations of the maximum transverse velocity $\mathrm{U}_{3 \max }$ according to different values of parameter of vortex viscosity (microrotation parameter) $K$ for N2 and N3. It is interesting to note that the non-micropolar nanofluid $(K=0)$ has a higher transverse velocity section compared to transverse micropolar nanofluid $(K \neq 0)$ independently of the type of nanofluid. In addition, this figure shows that the maximum transverse velocity decreases when $K$ increases for a nanoparticles'volume fraction fixed. As a result, the vortex viscosity setting reduces the three-dimensional character of the flow.

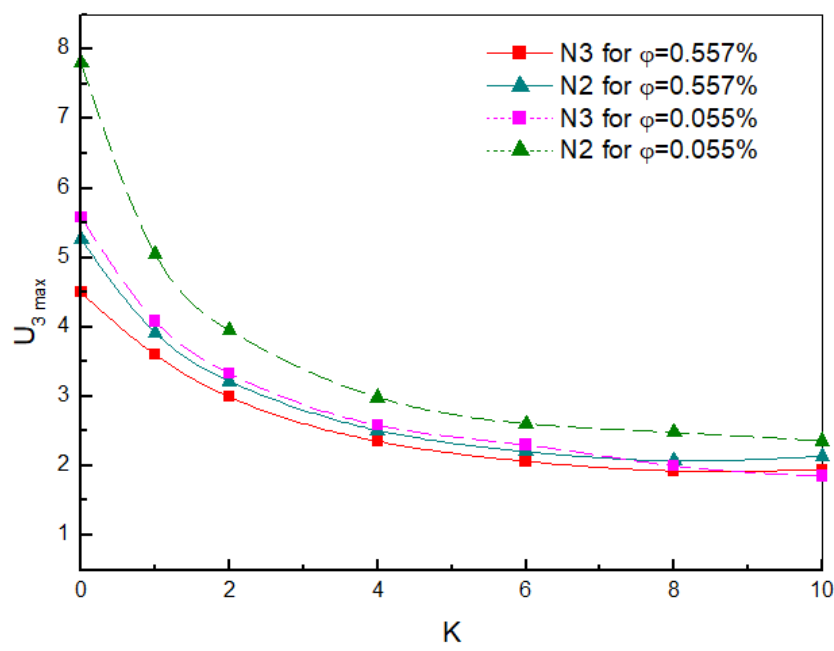

Fig. 20 Variation of the maximum transverse velocity $U_{3 \max }$ according the micropolar vortex parameter $\mathrm{K}$, for $\varphi=0.055 \%,=0.557 \%$ and $N=-0.2$

The respective variations of maximum values of the $\mathrm{x}$ and $\mathrm{y}$ components of the microrotation velocity namely $\mathrm{H}_{1 \max }$ and $\mathrm{H}_{2 \max }$ are shown in Fig. 21. The variations are plotted against the micropolar parameter for two values of nanoparticles volume fraction. As shown 
by this figure, for both $\mathrm{N} 2$ and $\mathrm{N} 3$, the $\mathrm{H}_{1 \max }$ and $\mathrm{H}_{2 \max }$ curves show a similar tendency. In addition, the values of the microrotation velocity increase with the enhancement of the micropolar parameter for both types of surfactant and regardless of nanoparticles volume fraction. When the micropolar parameter remains constant, the magnitudes of $\mathrm{H}_{1 \max }$ and $\mathrm{H}_{2 \max }$ tend to diminish as the volume fraction of nanoparticles is enhanced.
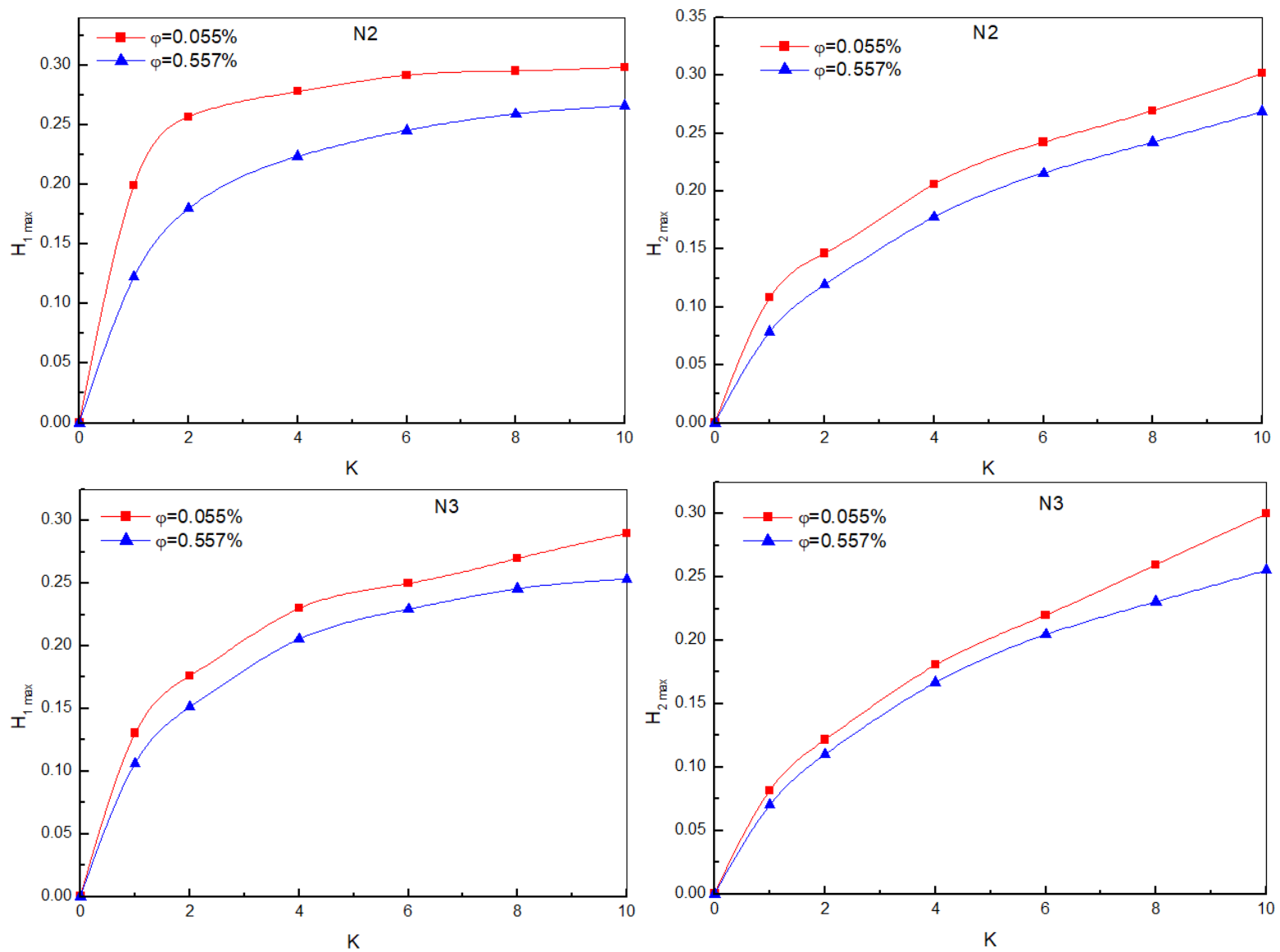

Fig. 21 Variation of the maximum of the components of microtation velocity $\mathrm{H}_{1}$ max and $\mathrm{H}_{2}$ max according to the micropolar vortex parameter $\mathrm{K}$, for $\varphi=0.055 \%, \varphi=0.557 \%, \mathrm{Ra}=10^{5} \mathrm{and}$

$$
N=-0.2
$$

\section{Conclusion}

Three-dimensional computational analysis has been performed toinvestigate the double diffusive natural convection of micropolar multi-walled carbon nanotubes-water nanofluids stabilized with two types of surfactants lignin and sodium polycarboxylatein a differentially heated cubical enclosure for different governing parameters (Rayleigh number, micropolar parameter, and nanoparticles volume fraction). This numerical work has been performed considering real experimental data for thermophysical properties of multi-walled carbon 
nanotubes-water nanofluids. The main findings of this numerical analysis can be summarized as follows

- The multi-walled carbon nanotubes-water nanofluid stabilized with lignin exhibits more heat and mass transfer rates when compared to multi-walled carbon nanotubes-water nanofluids stabilized with sodium polycarboxylate. So using lignin as a surfactant is better than using sodium polycarboxylate.

- Heat and mass transfer rates are lower for the micropolar nanofluid model when compared to the pure nanofluid model.

- Irrespective to the considered types of surfactant, the enhancement in the micropolar material parameter weakens both flow strength and three-dimensional character of the flow.

- The flow strength and the three-dimensional effect of the flow are ameliorated when the nanoparticle volume concentration is less than the critical valueand then are deteriorated when it exceed this value for both two types of nanofluids.

- Both average Nusselt $\overline{N u}$ and Sherwood $\overline{S h}$ numbers increase withan increase in Rayleigh number.

- Employing a nanofluid stabilized with Lignin ameliorates the average Nusselt and Sherwood numbers more than that containing sodium polycarboxylate.

- Using nanofluid is better than pure water solution when nanoparticles volume concentration is less than a critical value.

- The micropolar model can describe the impacts of the microstructures on fluid motion.

- The microrotation of the nanoparticles seems to play a significant roleinto flow regime and in heat and mass transfer rates.

\section{Acknowledgement:}

The authors extend their appreciation to the Deanship of Scientific Research at King Khalid University, Abha, Saudi Arabia for funding this work through the General Research Project under grant number (G.R.P-74-42).

The first author gratefully acknowledges the Tunisian Ministry of Higher Education and Scientific Research for financial support to her stay in LGCGM, France. 


\section{Nomenclature}

C

C'

$\mathrm{Cp}$

D

G

$\stackrel{\omega}{\mathrm{H}}$

$\stackrel{n}{\mathrm{H}^{\prime}}$

J

K

K

$k$

$\mathrm{L}$

Le

$\mathrm{N}$

$\mathrm{Nu}$

$\overline{N u}$

$P$

$\operatorname{Pr}$

$R a$

Sh dimensionless species concentration, $C=\frac{C^{\prime}-C_{l}^{\prime}}{C_{H}^{\prime}-C_{L}^{\prime}}$.

species concentration $\left(\mathrm{kgm}^{-3}\right)$

specific heat at constant pressure $\left(\mathrm{kJ} \mathrm{kg}^{-1} \mathrm{~K}^{-1}\right)$

species diffusivity $\left(\mathrm{m}^{2} \mathrm{~s}^{-1}\right)$

acceleration of gravity $\left(\mathrm{m} \mathrm{s}^{-2}\right)$

dimensionless microrotation vector

the microrotation vector $\left(\mathrm{m} \mathrm{s}^{-1}\right)$

micro-inertia coefficient (microrotation radius)

micropolarparameter, $K=\frac{k}{\mu_{f}}$

vortex viscosity $\left(\mathrm{kg} \mathrm{m}^{-1} \mathrm{~s}^{-1}\right)$

thermal conductivity $\left(\mathrm{W} \mathrm{m}{ }^{-1} \mathrm{~K}^{-1}\right)$

enclosure height(m)

Lewis number, $L e=\frac{\alpha}{D}$

buoyancy ratio, $N=\frac{\beta_{C f}\left(C_{H}-C_{L}\right)}{\beta_{T f}\left(T_{H}-T_{C}\right)}$

local Nusselt Number

average Nusselt Number

dimensionless pressure

Prandtl number, $\operatorname{Pr}=\frac{v_{f}}{\alpha_{f}}$,

thermal Rayleigh number, $R a=\frac{g \beta_{T f}\left(T_{H}-T_{C}\right) L^{3}}{v_{f} \alpha_{f}}$

local Sherwood number

average Sherwood number

dimensionless time, $t=\frac{\alpha t^{\prime}}{L^{2}}$ 
dimensionless temperature, $T=\frac{T^{\prime}-T_{C}^{\prime}}{T_{H}^{\prime}-T_{C}^{\prime}}$

T'

Temperature(K)

$\vec{U}$

dimensionless velocity $\vec{U}=\frac{\overrightarrow{U \prime L}}{\alpha_{f}}$

$\vec{U}^{\prime}$

$\operatorname{velocity}\left(\mathrm{m} \mathrm{s}^{-1}\right)$

$\mathrm{x}, \mathrm{y}, \mathrm{z}$

dimensionless Cartesian coordinates, $\mathrm{x}=\mathrm{x}^{\prime} / \mathrm{L}, \mathrm{y}=\mathrm{y}^{\prime} / \mathrm{L}, \mathrm{z}=\mathrm{z}^{\prime} / \mathrm{L}$

\section{Greek symbols}

$\alpha$

$\beta_{\mathrm{C}}$

$\beta_{\mathrm{T}}$

$\varphi$

$\mu$

$v$

$\rho$

$\mu$

$\vec{\omega}^{\prime}$

$\ddot{\psi}$

$\vec{\psi}^{\prime}$

\section{Subscripts}

C

$f$

$\mathrm{H}$

L thermal diffusivity $\left(\mathrm{m}^{2} \mathrm{~s}^{-1}\right)$

coefficient of compositional expansion $\left(\mathrm{m}^{3} \mathrm{~kg}^{-1}\right)$

coefficient of thermal expansion $\left(\mathrm{K}^{-1}\right)$

solid volume fraction

dynamic viscosity $\left(\mathrm{kgm}^{-1} \mathrm{~s}^{-1}\right)$

kinematic viscosity $\left(\mathrm{m}^{2} \mathrm{~s}^{-1}\right)$

density $\left(\mathrm{kg} \mathrm{m}^{-3}\right)$

dimensionless vorticity, $\vec{\omega}=\frac{\overrightarrow{\omega \prime} L}{\alpha_{f}}$

$\operatorname{vorticity}\left(\mathrm{s}^{-1}\right)$

dimensionless vector potential of velocity, $\vec{\psi}=\frac{\overrightarrow{\psi \prime}}{\alpha_{f}}$

vector potential of velocity $\left(\mathrm{m}^{2} \mathrm{~s}^{-1}\right)$

Cold

Fluid

Hot

Low 


$\begin{array}{ll}\text { max } & \text { Maximum } \\ \text { nf } & \text { Nanofluid } \\ \text { P } & \text { Particle } \\ 1 & \text { x-component } \\ 2 & \text { y-component } \\ 3 & \text { z-component }\end{array}$

\section{Superscripts}

dimensional variables

\section{References:}

[1] Putra N, Roetzel W, Das SK. Natural convection of nano-fluids.Heat Mass Transf. 2002; 39:775-784.

[2] Khanafer K, Vafai K, Lightstone M. Buoyancy-driven heat transfer enhancement in a twodimensional enclosure utilizing nanofluids. Int J Heat Mass Trans. 2003; 46: 3639-3653.

[3] Jou RY,Tzeng SC. Numerical research of nature convective heat transfer enhancement filled with nanofluids in rectangular enclosures. Comm Heat Mass Transf. 2006; 33: 727736.

[4] Abu-Nada E, Masoud Z, Hijazi A. Natural convection heat transfer enhancement in horizontal concentric annuli using nanofluids. IntComm Heat Mass Transf. 2008; 35: $657-665$.

[5] Oztop HF, Abu-Nada E. Numerical study of natural convection in partially heated rectangular enclosures filled with nanofluids. Int J Heat Fluid Flow. 2008; 29: 1326-1336.

[6] Minea AA. Uncertainties in modeling thermal conductivity of laminar forced convection heat transfer with water alumina nanofluids. Int J Heat Mass Transf. 2014; 68: 78-84.

[7] Bouhalleb M, Abbassi H. Numerical investigation of heat transfer by $\mathrm{CuO}-$ Water nanofluid in rectangular enclosures. Heat Transf Eng. 2015; 37: 13-23.

[8] Minea AA, Buonomo B, Burggraf J, Ercole D, Karpaiya KR, Di Pasqua A, Sekrani G, Steffens J, Tibaut J, Wichmann N, Farber P, Huminic A, Huminic G, Mahu R, Manca 
O,Oprea C, Poncet S, Ravnik J. NanoRound: A benchmark study on the numerical approach in nanofluids' simulation. Int Comm Heat Mass Transf. 2019; 108: 104292.

[9] Purusothaman A, Nithyadevi N, Oztop HF, Divya V, Al-Salem K. Three dimensional numerical analysis of natural convection cooling with an array of discrete heaters embedded in nanofluid filled enclosure. Adv Pow Tech. 2018; 27: 268-280.

[10] Selimefendigil F, Öztop HF. Mixed convection of nanofluids in a three dimensional cavity with two adiabatic inner rotating cylinders. Int J Heat Mass Transf. 2017; 117: 331-343.

[11] Esfahani AJ, Bordbar V. Double diffusive natural convection heat transfer enhancement in a square enclosure using nanofluids. J. Nano Technol Eng Med. 2011; 2: 021002-021011.

[12] Parvin S, Nasrin R, Alim MA, Hossain NF. Double diffusive natural convection in a partially heated enclosure using nanofluid. Heat Transf Asian Res J. 2012; 41; 484-497.

[13] Chen S, Yang B, Luo KH, Xiong X, Zheng C. Double diffusion natural convection in a square cavity filled with nanofluid. Int J Heat Mass Transf. 2016; 95: 1070-1083.

[14] Aly AM, Raizah ZAS. Double-diffusive natural convection in an enclosure filled with nanofluid using ISPH method. Alex Eng J. 2016; 55: 3037-3052.

[15] Wen D, Ding Y. Effective thermal conductivity of aqueous suspensions of carbon nanotubes (carbon nanotube nanofluids). J Thermal Heat Transf. 2004; 18: 481-485.

[16] Jiang W, Ding G, Peng H. Measurement and model on thermal conductivities of carbon nanotube nanorefrigerants. Int J Thermal Sci. 2009;48: 1108-1115.

[17] Halelfadl S, Adham AM, Mohd-Ghazali N, Maré T, Estellé P, Ahmad R.Optimization of thermal performances and pressure drop of rectangular microchannel heat sink using aqueous carbon nanotubes based nanofluid. App Thermal Eng. 2014; 62: 492-499.

[18] Estellé P, Halelfadl S, Maré T. Thermal conductivity of CNT water based nanofluids: Experimental trends and models overview. J Thermal Eng. 2015; 1: 381-390

[19] Estellé P, Halelfadl S, Maré T. Lignin as dispersant for water-based carbon nanotubes nanofluids:Impact on viscosity and thermal conductivity. Int Com Heat Mass Transf. 2014; 57: 8-12.

[20] Rahman MM, Öztop HF, Steele M, Naim A.G, Al-Salem K, Ibrahim TA. Unsteady natural convection and statistical analysis in a CNT-water filled cavity with nonisothermal heating. Int Com Heat Mass Transf. 2015; 64: 50-60. 
[21] Job VM, Gunakala SR. Unsteady MHD Free Convection Nanofluid Flows Within A Wavy Trapezoidal Enclosure With Viscous And Joule Dissipation Effects. Num Heat Transf, Part A. 2015; 59: 1-23.

[22] Estellé P, Mahian O, Maré T, Öztop HF. Natural convection of CNT water-based nanofluids in a differentially heated square cavity. J Thermal Anal Calorim. 2017; 28: $1765-1770$.

[23] Minea AA, Estellé P. Numerical study on CNT nanofluids behavior in laminar pipe flow. J Mol Liq. 2018; 271: 281-289.

[24] Kolsi L, Alrashed AAAA, Al-Salem K, Oztop HF, Borjini MN, Control of natural convection via inclined plate of CNT-water nanofluid in an open sided cubical enclosure under magnetic field. Int J Heat Mass Transf. 2017; 111: 1007-1018.

[25] Al-Rashed AAAA, Kolsi L, Kalidasan K, Malekshah EH, Borjini MN, Kanna PR. Second law analysis of natural convection in a CNT-Water nanofluid filled inclined 3D Cavity with incorporated Ahmed Body. Int J Mech Sci. 2017; 130: 399-415.

[26] Rahimi A, Kasaeipoor A, Malekshah EH, Kolsi L. Experimental and numerical study on heat transfer performance of three-dimensional natural convection in an enclosure filled with DWCNTs-water nanofluid. Powd Tech. 2017; 322:340-352.

[27] Eringen AC. Theory of micropolar fluids, J MathMech. 1966; 16: 1-18.

[28] Aydin O, Pop I. Natural convection from a discrete heater in enclosures with a micropolar fluid. Int J Eng Sci. 2015; 43; 1409-1418.

[29] Zadravec M, Hriberšek M, Škerget L. Natural Convection Of Micropolar Fluids in an Enclosure With Boundary Element Method. Eng Anal with Bound Elem. 2009; 33: 485492.

[30] Jena SK, Malla LK, Mahapatra SK, Chamkha AJ. Transient buoyancy-opposed double diffusive convection of micropolar fluids in a square enclosure. Int $\mathrm{J}$ Heat Mass Transf.2014;81: 681-694.

[31] Abidi A, Borjini MN. Effects of Microstructure on Three-Dimensional DoubleDiffusive Natural Convection Flow of Micropolar Fluid. Heat Transf Eng. 2019; 41: 361376.

[32] Bourantas GC, Loukopoulos VC. Modeling the natural convective flow ofmicropolar nanofluids. Int J Heat Mass Transf. 2014; 68: 35-41. 
[33] Hashemi H, Namazian Z, Mehryan SAM. Cu-water micropolar nanofluid natural convection within a porous enclosure with heat generation. J Mol Liq. 2017; 236: 48-60.

[34] Hussanan A, Salleh MZ, Khan I, Shafie S. Convection heat transfer in micropolar nanofluids with oxide nanoparticles in water, kerosene and engine oil. J MolLiq. 2017; 229: 482-488.

[35] Bourantas GC, Loukopoulos VC. MHD natural-convection flow in an inclined square enclosure filled with a micropolar-nanofluid. Int J Heat MassTransf. 2014; 79: 930-944.

[36] Ahmed SE, Mansour MA, Hussein AK, Sivasankaran S. Mixed convection from a discrete heat source in enclosures with two adjacent moving walls and filled with micropolar nanofluids. Eng SciTechInt J. 2016; 19: 364-376.

[37] Nering K, Rup K.The effect of nanoparticles added to heated micropolar fluid, Superlattices and Microstructures. 2016; 98:283-294.

[38] Izadi M, Mehryan SAM, Sheremet MA.Natural convection of CuO-water micropolar nanofluids inside a porous enclosure using local thermal non-equilibrium condition. $\mathrm{J}$ Taiwan Institute Chem Eng. 2018; 88: 89-103.

[39] Rashad A, Mansour M, Gorla RSR. Mixed convection from a discrete heater in liddriven enclosures filled with non-Newtonian nanofluids. J Nanomaterials, Nanoengineering and Nanosystems. 2017; 231: 3-16.

[40] Abidi A, Raizah Z, Madiouli J. Magnetic Field Effect on the Double Diffusive Natural Convection in Three-Dimensional Cavity Filled with Micropolar Nanofluid. App Sci. 2018; 8: 2342-2367.

[41] Manaa N, Abidi A, Saleel CA, Al Makwash SM, Borjini MN. On Simulation of Double-Diffusive Natural Convection in a Micropolar Nanofluid Filled Cubic Cavity. Heat Transf Eng. 2020; https://doi.org/10.1080/01457632.2020.1756074.

[42] Manaa N, Abidi A, Ahamed SC, Madiouli J, Borjini MN. Three-Dimensional Numerical Analysis on Performance Enhancement of Micropolar Hybrid Nanofluid in Comparison with Simple Nanofluid. Heat Transf Eng. 2020; https://doi.org/10.1080/01457632.2020.1807106.

[43] Therme-Excel: Physical characteristics of water at the atmospheric pressure. Last update: 2003.http://www.thermexcel.com/english/tables/eau_atm.htm. 
[44] Mohd-Ghazali N, Estellé P, Halelfadl S, Maré T, Siong TC, Abidin U. Thermal and hydrodynamic performance of a microchannel heat sink with carbon nanotube nanofluids. J Them Anal Calorim. 2019; 138: 937-945.

[45] Agarwal RS, Bhargava R, Balaji AVS.Finite element solution of non-steady three dimensional micropolar fluid flow at a stagnant point. Int J Eng Sci. 1990; 28: 851-857.

[46] Takhar HS, Agarwal RS, Bhargava R, Jain S. Mixed convective non-steady 3dimensional micropolar fluid flow at a stagnation point. Heat Mass Transf J. 1998; 33: 443-448.

[47] Chamkha AJ, Jaradat M, Pop I. Three dimensional micropolar flow due to a stretching flat surface. Int J Fluid Mech Res. 2003; 30: 357-366.

[48] Guram GS, Smith C. Stagnation flows of micropolar fluids with strong and weak interactions, Comp Math App; 1980;6: 213-233.

[49] Sezai I, Mohamad AA. Double diffusive convection in a cubic enclosure with opposing temperature and concentration gradient. Phys Fluids. 2000; 12: 2210-2223.

[50] Kolsi L, Hussein AK, Borjini MN, Mohammed HA, Aïssia HB. Computational Analysis of Three-Dimensional Unsteady Natural Convection and Entropy Generation in a Cubical Enclosure Filled with Water- $\mathrm{Al}_{2} \mathrm{O}_{3}$ Nanofluid. Arab J Sci Eng. 2014; 39:74837493. 